\title{
On eigenfunctions of Markov processes on trees
}

\author{
Laurent Miclo \\ Laboratoire d'Analyse, Topologie, Probabilités, U.M.R. 6632 \\ Université de Provence and C.N.R.S. \\ France
}

\begin{abstract}
We begin by studying the eigenvectors associated to irreducible finite birth and death processes, showing that the $i^{\text {th }}$ nontrivial eigenvector $\varphi_{i}$ admits a succession of $i$ decreasing or increasing stages, each of them crossing zero. Imbedding naturally the finite state space into a continuous segment, one can unequivocally define the zeros of $\varphi_{i}$, which are interlaced with those of $\varphi_{i+1}$. These kind of results are deduced from a general investigation of minimax multi-sets Dirichlet eigenproblems, which leads to a direct construction of the eigenvectors associated to birth and death processes. This approach can be generically extended to eigenvectors of Markov processes living on trees. This enables to reinterpret the eigenvalues and the eigenvectors in terms of the previous Dirichlet eigenproblems and a more general conjecture is presented about related higher order Cheeger inequalities. Finally, we carefully study the geometric structure of the eigenspace associated to the spectral gap on trees.
\end{abstract}

Keywords: birth and death processes, Markov processes on trees, eigendecomposition of generators, Dirichlet eigenproblems, isospectral partition, nodal domains, Cheeger inequalities, spectral gap.

MSC2000: first: 60J80, secondary: 15A18, 49R50, 26A48. 


\section{Introduction and results}

The official purpose of this paper is to give a description of the shape of the eigenvectors associated to finite birth and death processes. Indeed, this subject is quite classical, because generators of finite irreducible birth and death processes are totally positive matrices (up to the addition of a factor of the identity, this follows from results in a paper of Karlin and McGregor [14], for instance) for which a whole theory has been developped (see the book of Karlin [15] for an extensive account or the article of Fomin and Zelevinsky [10] for a more friendly introduction, and the references given therein), starting in the 1930's with the works of Schoenberg [19] and Gantmacher and Krein [13]. In the latter article, the authors showed in particular that the eigenvectors associated to the $k^{\text {th }}$ eigenvalue have exactly $k-1$ sign changes, for $1 \leq k \leq N$, with $N$ the size of the matrix. The corresponding question in a continuous framework is even better known, since it is related to the famous Courant theorem for Sturm-Liouville operators, see for instance the section VI.6 of [7]. Nevertheless, we would like to give a rather simple and probability-oriented (even if the techniques will remain rather analytical) proof of the results presented below. We did not find in the litterature those concerning monotonicity properties, so in fact we do not know if they hold more generally for totally positive matrices. Furthermore, some of the presented arguments and behaviors can be extended to Markov processes on trees, a situation which is outside the scope of total positivity (except for the path case), but here we will only begin such an investigation.

Maybe more important is the unofficial message we would like to convey about some quantities $\Lambda_{k}$ we introduce in next section for general reversible (finite) Markov processes, since we believe they could bring insight to more difficult questions, specially those concerning higher order Cheeger inequalities. Another motivation for the consideration of the $\Lambda_{k}$, is that they may give some clues about a probabilistic interpretation of the eigenvalues in the setting of birth and death processes, concerning their relations with strong stationary times (see Theorem 4.20 of Diaconis and Fill [9]). But coming back to the object of this paper, we will see that the results presented below are consequences of the first properties one can deduce for the $\Lambda_{k}$.

So we consider an irreducible birth and death process on the state space $V:=\{0, \ldots, N\}$. The simplest way to specify it is through its jump rates $b_{x}>0$ from $x$ to $x+1$, for $0 \leq x<N$, and $d_{x}>0$, from $x$ to $x-1$, for $0<x \leq N$ (it is also convenient to define $b_{N}=d_{0}=0$ ). The corresponding generator $L$, acting on $\mathcal{F}(V)$, the space of real-valued functions defined on $V$, is given by

$$
\forall f \in \mathcal{F}(V), \forall x \in V, \quad L[f](x):=b_{x}(f(x+1)-f(x))+d_{x}(f(x-1)-f(x))
$$

It is well-known that $L$ admits a unique invariant probability $\pi$. It is indeed reversible, meaning that $L$ is self-adjoint in $\mathbb{L}^{2}(\pi)$. So let $\lambda_{0} \leq \lambda_{1} \leq \ldots \leq \lambda_{N}$ the eigenvalues of $-L$, in increasing order. It is easy to check that $\lambda_{0}=0$, with eigenspace $\operatorname{Vect}(\mathbb{1})$, and that all the eigenvalues have multiplicity one, so we have $0=\lambda_{0}<\lambda_{1}<\cdots<\lambda_{N}$. Let $\varphi_{0}, \varphi_{1}, \ldots, \varphi_{N}$ be some corresponding (non null) eigenvectors. If for some $0 \leq k \leq N$, we have $\varphi_{k}(0)=0$, then using the relation $L_{k}\left[\varphi_{k}\right](x)=\lambda_{k} \varphi_{k}(x)$ for $x$ going from the left of $V$ to the right, we would get that $\varphi_{k} \equiv 0$ (one would have noticed that this argument also shows the above multiplicity one assertion). Thus we can normalize the $\varphi_{k}$, for $0 \leq k \leq N$, with the unusual convention that $\varphi_{k}(0)=1$.

Let us recall that a nodal domain of a function $\varphi \in \mathcal{F}(V)$ is a connected component (with respect to the usual graph structure of $V$ ) of the set $\{x \in V: \varphi(x) \neq 0\}$. One of the results from the paper of Gantmacher and Krein [13] we will recover, is that for any fixed $0 \leq k \leq N, \varphi_{k}$ admits exactly $k+1$ nodal domains. Let us denote them by $A_{k, 0}, A_{k, 1}, \ldots, A_{k, k}$, ordered by their smallest elements. As can be guessed, $\varphi_{k}$ is positive or negative on $A_{k, i}$, according to the parity of $i$. But one can be more precise: write $A_{k, i}:=\llbracket a_{k, i}^{-}, a_{k, i}^{+} \rrbracket$. Then for $i$ between 0 and $k-1$, we have that

$a_{k, i+1}^{-}-a_{k, i}^{+}$is either equal to 1 or 2 , i.e. there is at most one point between $A_{k, i}$ and $A_{k, i+1}$, where $\varphi_{k}$ vanishes. In case such a point exists, let us denote it by $c_{k, i}$. If it does not exist, we introduce 
a new virtual point $c_{k, i}$ belonging to the continuous interval $[0, N]$, but not to $V$, in the following way: we extend $\varphi_{k}$ into a function $\bar{\varphi}_{k}$ on $[0, N]$ so that it is affine on any of the intervals $[l, l+1]$, for $0 \leq l<N$, and we take $c_{k, i}$ the unique point in $\left(a_{k, i}^{+}, a_{k, i+1}^{-}\right)$where $\bar{\varphi}_{k}$ vanishes. Then we will prove the following interlacing property for the finite sequences $\left(c_{k, i}\right)_{0 \leq i<k}$ :

$$
\forall 0<k<N, \forall 0 \leq i<k, \quad c_{k+1, i}<c_{k, i}<c_{k+1, i+1}
$$

Finally, we describe some monotonicity properties of the eigenvectors $\varphi_{k}$. For $0<k \leq N$ and $0<i<k$, one can introduce in a unique way two successive or equal point(s) $e_{k, i}^{-}, e_{k, i}^{+}$in $A_{k, i}$, so that, if $k$ is odd (respectively even) $\varphi_{k}$ is decreasing (resp. increasing) on $\llbracket e_{k, i-1}^{+}, e_{k, i}^{-} \rrbracket$, increasing (resp. decreasing) on $\llbracket e_{k, i}^{+}, e_{k, i+1}^{-} \rrbracket$ and $\varphi_{k}\left(e_{k, i}^{-}\right)=\varphi_{k}\left(e_{k, i}^{+}\right)$. For the left hand and right hand domains, we have that $\varphi_{k}$ is decreasing on $\llbracket 0, e_{k, 1}^{-} \rrbracket$ and increasing on $\llbracket e_{k, k-1}^{+}, N \rrbracket$ if $k$ is even and decreasing otherwise (for $k=0$, this does not hold, since remember that $\varphi_{0}=\mathbb{1}$ ).

Generically, the above behaviors can be simplified, since we have $c_{k, i} \notin V$ and $e_{k, i}^{-}=e_{k, i}^{+}$, for $0<i<k \leq N$. To give a rigorous result, these statements hold almost surely (a.s.) if the birth and death rates $b_{x}$, for $0 \leq x<N$, and $d_{x}$, for $0<x \leq N$ are sampled independently according to laws absolutely continuous with respect to the restriction of the Lebesgue measure on $(0,+\infty)$. Nevertheless, one should keep in mind the prototype of the simple random walk on $V$ (for its usual nearest neighbour graph structure) for these behaviors, where for $0 \leq k \leq N$, the above eigenvectors $\varphi_{k}$ are given by the restrictions of the functions $\cos (\pi k \cdot / N)$ to $V$ (they correspond to the eigenvalues $\left.\lambda_{k}=1-\cos (\pi k / N)\right)$.

These results can be extended to one-dimensional continuous frameworks, the proofs even simplify if the setting is sufficiently regular, for instance for elliptic reflected diffusions on compact intervals, with smooth coefficients. But one can expect a general formulation via Dirichlet forms which includes all cases (discrete or continuous irregular situations), see [17] where this was done for the spectral gap, but we will not enter into the associated technicalities here. In discrete case or for regular diffusions, the monotonicity of the eigenvectors associated to the spectral gap was also obtained by Chen and Wang, respectively in [5] and in $[4,6]$.

The article will be organised according to the following plan. In the next section we will develop a Dirichlet eigenproblem point of view for general finite irreducible generators and show that it is convenient to extend the state space into a continuous graph, in the spirit of the geometric realizations considered by Friedman [11] for simple random walks. We will take advantage of this exposition to present an interesting conjecture about higher order Cheeger inequalities, which later in the paper will be proven to be true for generators whose underlying graph is a tree. Coming back to birth and death processes in section 3, these considerations will enable us to construct the eigenvectors directly. The underlying principle can be translated into a numerical algorithm, but we will not study here its efficiency. The behaviors mentioned in this introduction will be deduced from this construction and in particular we will recover the nodal theorem for birth and death processes. The fourth section will deal with the a.s. assertions and we will also see that the previous constructions can be generically extended to processes whose associated graph is a tree. In last section we will investigate the shape of the eigenvectors corresponding to the spectral gap on trees, exhibing a center point and certain monotonicity features.

\section{Dirichlet eigenproblems}

Here we will work in the general setting of finite irreducible and reversible Markov processes. We introduce for them some quantities which are believed to be close to the eigenvalues. They combine a spectral feature, since they are related to first Dirichlet eigenvalues associated to subdomains, with a kind of multi-isoperimetry, where sets play an important role. In the particular cases when 
the underlying graph is a tree, the quantities introduced coincide exactly with the eigenvalues, but this is not true in general.

Still with the state space $V=\{0,1, \ldots, N\}$, we are given a generator $L=(L(x, y))_{x, y \in V}$ assumed to be irreducible and reversible with respect to some probability $\pi$, which is then positive on $V$. We endow the latter set an unoriented graph structure, by saying that for $x, y \in V,\{x, y\}$ is an edge if and only if $L(x, y)>0$ (which is equivalent to $L(y, x)>0$ by reversibility, also note there are no loops). We will denote by $E$ the collection of these edges. For our purpose, it is better to see this graph $(V, E)$ as a continuous space $\bar{V}$, where each edge $\{x, y\} \in E$ is replaced by an edge-segment, written $[x, y]$, of length $1 /(\pi(x) L(x, y))$. Of course the boundary points of these edge-segments corresponding to a given vertex $x \in V$ are all identified with a unique point still designated by $x$, so $V$ is naturally embedded into $\bar{V}$. Sometimes we will refer to the elements of $\bar{V} \backslash V$ as virtual points. On each edge-segment $[x, y]$ we use classical calculus with respect to the length parameter, in particular we consider the natural Lebesgue measure $\lambda_{[x, y]}$ and the whole space $\bar{V}$ is endowed with the measure $\lambda:=\sum_{\{x, y\} \in E} \lambda_{[x, y]}$. The formula $\pi=\sum_{x \in V} \pi(x) \delta_{x}$ also enables us to extend $\pi$ to $\bar{V}$. Such a continuous setting was introduced by Friedman [11] for simple random walks (where $L(x, y)=1$ for any $\{x, y\} \in E)$.

Coming back to $V$, let us recall that the Dirichlet form $\mathcal{E}$ associated to $\pi$ and $L$ is given by

$$
\begin{aligned}
\forall f \in \mathcal{F}(V), \quad \mathcal{E}(f) & :=-\pi(f L[f]) \\
& =\frac{1}{2} \sum_{x, y \in V} \pi(x) L(x, y)(f(y)-f(x))^{2}
\end{aligned}
$$

To extend this notion to $\bar{V}$, we denote by $\mathcal{F}(\bar{V})$ the space of absolutely continuous functions on $\bar{V}$ (i.e. that are absolutely continuous on all edge-segments) and for any $f \in \mathcal{F}, \overline{\mathcal{F}}(f)$ will designate the subspace of functions $F \in \mathcal{F}(\bar{V})$ which coincide with $f$ on $V$. Then we define a Dirichlet form $\overline{\mathcal{E}}$ on $\mathcal{F}(\bar{V})$ by taking

$$
\forall F \in \mathcal{F}(\bar{F}), \quad \overline{\mathcal{E}}(F):=\int\left(F^{\prime}\right)^{2} d \lambda \in \overline{\mathbb{R}}_{+}
$$

where $F^{\prime}$ stands for the weak derivative of $F$. Rigorously speaking, the Dirichlet form should be the restriction of $\overline{\mathcal{E}}$ to $\mathcal{D}(\overline{\mathcal{E}}):=\{F \in \mathcal{F}(\bar{V}): \overline{\mathcal{E}}(F)<+\infty\}$ and note that it is not naturally defined on the space $\mathbb{L}^{2}(\pi)$, so the classical theory of Dirichlet forms (cf. for instance the book of Fukushima, Osshima and Takeda [12]) cannot be applied to associate to $(\pi, \overline{\mathcal{E}})$ a regular Markov process (on this subject, see also the end of Remark 1 below). Nevertheless, the link with the previous discrete Dirichlet form is that

$$
\forall f \in \mathcal{F}, \quad \mathcal{E}(f)=\min _{F \in \overline{\mathcal{F}}(f)} \overline{\mathcal{E}}(F)
$$

as can be easily checked, the minimizing $F \in \mathcal{F}(f)$ is the affine extension of $f$ on each of the edge-segments of $\bar{V}$.

We now introduce the first Dirichlet eigenvalue associated to a subdomain, first in $V$, so we can recall its probabilistic interpretation. Let $A \subset V$, the corresponding first Dirichlet eigenvalue is defined as the quantity

$$
\lambda_{0}(A):=\inf _{f \in \mathcal{F}_{0}(A) \backslash\{0\}} \frac{\mathcal{E}(f)}{\pi\left(f^{2}\right)}
$$

where $\mathcal{F}_{0}(A)$ is the subspace of $\mathcal{F}(V)$ consisting of functions vanishing outside $A$ (the usual convention $\inf \emptyset=+\infty$ is assumed to enforced in the whole paper). In particular, we have $\lambda_{0}(V)=\lambda_{0}=0$. To see its meaning from a probabilistic point of view, let $\left(X^{(x)}(t)\right)_{t \geq 0}$ be a jump process on $V$, of generator $L$ and starting from $x \in V$. The first exit time from $A$ is defined by

$$
\tau_{A}^{(x)}:=\inf \left\{t \geq 0: X^{(x)}(t) \notin A\right\} \in \overline{\mathbb{R}}_{+}
$$


and it is well-known (cf. for instance the book manuscript of Aldous and Fill [1], chapter 3) that

$$
\lambda_{0}(A)=-\sup _{x \in A} \lim _{t \rightarrow+\infty} \frac{1}{t} \ln \left(\mathbb{P}\left[\tau_{A}^{(x)}>t\right]\right)
$$

If $A$ is assumed to be connected (with respect to the graph $(V, E)$ ), then the r.h.s. limit does not depend on the choice of $x \in A$ and $1 / \lambda_{0}(A)$ is a measurement of the difficulty to get out of $A$ for Markov processes of generator $L$.

In the same way, replacing $\mathcal{E}$ by $\overline{\mathcal{E}}$ and considering $\overline{\mathcal{F}}_{0}(A)$, the subspace consisting of functions from $\mathcal{F}(\bar{V})$ vanishing outside $A$, we can define $\bar{\lambda}_{0}(A)$, the first Dirichlet eigenvalue of a subset $A \subset \bar{V}$. But we will only be interested in subsets $A$ which are open, connected and whose intersection with $V$ is non empty. Call $\mathcal{A}_{0}$ the collection of all such subsets.

Remark 1 It is still possible to give an easy probabilistic interpretation of $\bar{\lambda}_{0}(A)$ for $A \in \mathcal{A}_{0}$, but one has to resort to instantaneous points (the considerations below are related to the framework of graph with boundary in the terminology of Friedman [11]).

So let $A \in \mathcal{A}_{0}$ be given and denote by $\partial A$ its topological boundary. We define a new generator $\widetilde{L}_{A}$ on $\widetilde{A}:=(A \cap V) \sqcup \partial A$ by first taking

$$
\forall x \neq y \in \widetilde{A} \cap V, \quad \widetilde{L}_{A}(x, y):=L(x, y)
$$

Next consider $z \in \widetilde{A} \backslash V$, then $z \in \partial A$ and there exists an edge $\{x, y\} \in E$ such that $z \in(x, y)$ (the interior of the edge-segment $[x, y]$ ) and let $x$ be choosen such that $[x, z) \subset A$. We define $\widetilde{L}_{A}(x, z):=1 /(\pi(x) \lambda([x, z]))$. In the unusual situation where $A \cap[x, y]=[x, y] \backslash\{z\}$, one has also to define $\widetilde{L}_{A}(y, z):=1 /(\pi(y) \lambda([y, z]))$, but apart from that case, we take $\widetilde{L}_{A}(v, z):=0$ for any other $v \in \widetilde{A} \cap V$. The values of $\widetilde{L}_{A}(z, v)$ for $z \in \widetilde{A} \backslash V$ and $v \in \widetilde{A}$ are not important for our purpose, because we need to consider a Markov process $\left(\widetilde{X}_{A}^{(x)}(t)\right)_{t \geq 0}$ on $\widetilde{A}$, associated to generator $\widetilde{L}_{A}$ and starting from $x \in \widetilde{A} \cap V$, only up to the exit time from $V \cap A$,

$$
\widetilde{\tau}_{A}^{(x)}:=\inf \left\{t \geq 0: X^{(x)}(t) \notin \widetilde{A} \cap V\right\} \in \overline{\mathbb{R}}_{+}
$$

Then, taking into account (2) and (3) applied to the probability $\widetilde{\pi}_{A}$, which is the restriction of $\pi$ to $A \cap V$ divided by $\pi(V \cap A)$, and to the Dirichlet form $\widetilde{\mathcal{E}}_{A}$ given by

$$
\begin{aligned}
\forall f \in \mathcal{F}(\widetilde{A}), \quad \widetilde{\mathcal{E}}_{A}(f):= & \frac{1}{2} \sum_{x, y \in A \cap V} \widetilde{\pi}_{A}(x) L(x, y)(f(y)-f(x))^{2} \\
& +\sum_{x \in \widetilde{A} \cap V, z \in \widetilde{A} \backslash V} \widetilde{\pi}_{A}(x) \widetilde{L}_{A}(x, z)(f(z)-f(x))^{2}
\end{aligned}
$$

it appears that for any $x \in \widetilde{A} \cap V$,

$$
\bar{\lambda}_{0}(A)=-\lim _{t \rightarrow+\infty} \frac{1}{t} \ln \left(\mathbb{P}\left[\widetilde{\tau}_{A}^{(x)}>t\right]\right)
$$

In fact, if one is interested in extending the generator $\widetilde{L}_{A}$ to the state space $V \sqcup(\widetilde{A} \backslash V)$, one should take for any $z \in \widetilde{A} \backslash V$, say $z \in(x, y)$ with $\{x, y\} \in E$,

$$
\forall v \in V \cup(\widetilde{A} \backslash\{z\}), \quad \widetilde{L}_{A}(z, v):= \begin{cases}+\infty & , \text { if } v=x \text { or } v=y \\ 0, & \text { otherwise }\end{cases}
$$

This means that $z$ is an instanteneous point for a Markov process $\left(\widetilde{X}_{A}(t)\right)_{t \geq 0}$ "associated" to this extended $\widetilde{L}_{A}$ : once it reaches $z$, it immediately jumps out of it, either to $x$ or $y$. The probability of going to $x$ (respectively $y$ ) should be $\lambda^{-1}([x, z]) /\left(\lambda^{-1}([x, z])+\lambda^{-1}([y, z])\right.$ ) (resp. $\left.\lambda^{-1}([x, y]) /\left(\lambda^{-1}([x, z])+\lambda^{-1}([y, z])\right)\right)$. Thus a jump from $x$ to $z$ should be interpreted as an attempt 
to jump from $x$ to $y$, which is accepted with probability $\lambda^{-1}([x, y]) /\left(\lambda^{-1}([x, z])+\lambda^{-1}([y, z])\right)$. Of course such a process can be constructed directly, but it cannot be characterized in the usual way through a generator matrix and its trajectories are not (a.s.) right continuous because of the instanteneous jumps. In some sense it is also reversible with respect to $\pi$, even if we have $\pi(\widetilde{A} \backslash V)=0$ (see also the above definition of the Dirichlet form $\widetilde{\mathcal{E}}_{A}$ ). Our initial process $(X(t))_{t \geq 0}$ can be recovered from $\left(\widetilde{X}_{A}(t)\right)_{t \geq 0}$ by erasing the instantaneous positions at times $t$ where $\widetilde{X}_{A}(t) \notin V$ and replacing them by the corresponding right limits. This construction can be done on any state space included in $\bar{V}$ which is a finite extension of $V$ and which contains at most one point in each $(x, y)$, for $\{x, y\} \in E$. If we add more points on such intervals, it is less obvious how to describe the transition between the (space-successive) instantaneous points. It seems the latter should be replaced by instantaneous excursions, but then one is led to get out of the state space $\bar{V}$. If it was possible to find a limit procedure through finer and finer discretizations of $\bar{V}$ containing $V$, one can imagine that the resulting (quite irregular) object would be a Markov process associated to $(\pi, \overline{\mathcal{E}})$. But this would not be the Brownian motion on $\bar{V}$, corresponding to $(\lambda / \lambda(\bar{V}), \overline{\mathcal{E}})$.

We are now going to consider pseudo-partitions of $\bar{V}$ made of elements from $\mathcal{A}_{0}$. So fix $0 \leq k \leq N$ and denote by $\mathcal{A}_{k}$ the set of disjoint $(k+1)$-tuples $A:=\left(A_{0}, \ldots, A_{k}\right)$ from $\mathcal{A}_{0}$. For such an element $A \in \mathcal{A}_{k}$, we define

$$
\Lambda(A):=\max _{0 \leq i \leq k} \bar{\lambda}_{0}\left(A_{i}\right)
$$

and

$$
\Lambda_{k}:=\inf _{A \in \mathcal{A}_{k}} \Lambda(A)
$$

which we will concentrate on here. Of course for $k=0$, we have $\Lambda_{0}=\bar{\lambda}_{0}(\bar{V})=0$. Also remark that since $\bar{\lambda}_{0}(\emptyset)=+\infty$, the above definition of $\Lambda_{k}$ would not have been modified if we had removed the requirement that the elements of $\overline{\mathcal{A}}_{0}$ should have nonempty intersection with $V$. This shows that the finite sequence $\left(\Lambda_{k}\right)_{0 \leq k \leq N}$ is nondecreasing.

To start an investigation of these quantities, we check that all the infima entering into their definitions are in fact attained. Next two lemmas are classical (see for instance Friedman [11]), we give them for the sake of completeness.

Lemma 2 For any $A \in \mathcal{A}_{0}$, there exists a unique function $F_{A} \in \overline{\mathcal{F}}_{0}(A)$, satisfying $\overline{\mathcal{E}}\left(F_{A}\right) / \pi\left(F_{A}^{2}\right)=$ $\bar{\lambda}_{0}(A)$ and normalized by $\max F_{A}=1$.

The function is positive on $A$ and satisfies on $A \cap V$ (but not on $\widetilde{A}$ ),

$$
\forall x \in A \cap V, \quad \widetilde{L}_{A}\left[f_{\widetilde{A}}\right](x)=-\bar{\lambda}_{0}(A) f_{\widetilde{A}}(x)
$$

where $\widetilde{A}$ and $\widetilde{L}_{A}$ are the set and the generator defined in Remark 1 and where $f_{\widetilde{A}}$ is the restriction to $\widetilde{A}$ of $F_{A}$.

\section{Proof}

By definition of $\overline{\mathcal{E}}$, in the infimum defining $\bar{\lambda}_{0}(A)$ it is sufficient to consider functions from $\overline{\mathcal{F}}_{0}(A)$ which are affine on any segment $[x, y]$ with $x \neq y \in \widetilde{A}$ and which is included in some edgesegment. Since such functions vanish outside $A$ and in particular on $\partial_{A}$, they are parametrized by their values on the finite set $A \cap V$. We can also restrict our attention to nonnegative functions, because for any $F \in \mathcal{F}(\bar{V})$, we have $\overline{\mathcal{E}}(|F|) \leq \overline{\mathcal{E}}(F)$ (and by irreducibility, this inequality is strict if $F$ has both positive and negative values). and of course $\pi\left(|F|^{2}\right)=\pi\left(F^{2}\right)$. Finally by homogeneity, we can enforce the normalisation asking for the functions to have their maximum equal to 1 . Thus we are led to the minimization of a continuous functional over the compact set 
$\left\{f \in \mathcal{F}(A \cap V): 0 \leq f \leq 1\right.$ and $\left.\max _{A \cap V} f=1\right\}$ and the first assertion follows, except for the uniqueness statement. Next let $F_{A} \in \overline{\mathcal{F}}_{0}(A)$ be a nonnegative minimizer and denote by $f_{\widetilde{A}}$ its restriction to $\widetilde{A}$. Of course $f_{\widetilde{A}}$ has to vanish on $\partial A$, but applying an usual variational argument to the value of $f_{\widetilde{A}}(x)$ for given $x \in A \cap V$, we get the relation mentioned in the lemma. It can be rewritten on $A \cap V$ as

$$
\widehat{L}_{A}\left[f_{A \cap V}\right]=-\bar{\lambda}_{0}(A) f_{A \cap V}
$$

where $f_{A \cap V}$ is the restriction of $F_{A}$ to $A \cap V$ and where the operator $\widehat{L}_{A}$ is defined on $\mathcal{F}(A \cap V)$ by

$$
\forall f \in \mathcal{F}(A \cap V), \forall x \in A \cap V, \quad \widehat{L}_{A}[f](x)=\sum_{y \in A \cap V} L(x, y)(f(y)-f(x))-f(x) \sum_{y \in \partial A} \widetilde{L}_{A}(x, y)
$$

The matrix associated to $-\widehat{L}_{A}$ is irreducible and all its off-diagonal entries are nonnegative, so Perron-Frobenius theorem we can be applied to see it that it admits a largest eigenvalue, which is of multiplicity one and whose eigenspace is generated by a positive function. Even more precisely, if an eigenfunction of $\widehat{L}_{A}$ is nonnegative, then it is indeed positive and associated to the largest eigenvalue. As a consequence, $\bar{\lambda}_{0}(A)$ is the largest eigenvalue of $\widehat{L}_{A}$ and its eigenspace is generated by $f_{A \cap V}$ and this ends the proof of the above lemma.

As a consequence of the above caracterization of minimizing functions, we get a simple but very useful monotonicity property for $\bar{\lambda}_{0}$ on $\mathcal{A}_{0}$.

Lemma 3 For any $A, B \in \mathcal{A}_{0}$, we have

$$
A \varsubsetneqq B \Longrightarrow \bar{\lambda}_{0}(A)>\bar{\lambda}_{0}(B)
$$

\section{Proof}

The inequality $\bar{\lambda}_{0}(A) \geq \bar{\lambda}_{0}(B)$ comes from $\overline{\mathcal{F}}_{0}(B) \subset \overline{\mathcal{F}}_{0}(A)$ and it is strict because of Lemma 2 : $F_{A}$ cannot be a minimizer for $\bar{\lambda}_{0}(B)$ because it is not positive on $B$ (notice that the openness and connectedness of the elements of $\mathcal{A}_{0}$ is crucial for the strict inequality).

Next we consider the second minimum previously introduced.

Lemma 4 For $0 \leq k \leq N$, there exists $A^{(k)}:=\left(A_{0}^{(k)}, \ldots, A_{k}^{(k)}\right) \in \mathcal{A}_{k}$ such that $\Lambda_{k}=\Lambda\left(A^{(k)}\right)$.

\section{Proof}

For fixed $0 \leq k \leq N$, choose a minimizing sequence from $\mathcal{A}_{k}$ and call it $\left(A^{(n, k)}\right)_{n \in \mathbb{N}}$ :

$$
\lim _{n \rightarrow \infty} \Lambda\left(A^{(n, k)}\right)=\Lambda_{k}
$$

Without loss of generality, we can also assume that for any $n \in \mathbb{N}, \Lambda\left(A^{(n, k)}\right) \leq 1+\Lambda_{k}$. For $n \in \mathbb{N}$, denote $A^{(n, k)}:=\left(A_{0}^{(n, k)}, \ldots, A_{k}^{(n, k)}\right)$ and for $0 \leq l \leq k, F_{n, l}:=F_{A_{l}^{(n, k)}}$. These functions satisfy

$$
\begin{aligned}
\lambda\left(\left(F_{n, l}^{\prime}\right)^{2}\right) & =\overline{\mathcal{E}}\left(F_{n, l}\right) \\
& \leq \Lambda\left(A^{(n, k)}\right) \mu\left(\left(F_{n, l}\right)^{2}\right) \\
& \leq 1+\Lambda_{k}
\end{aligned}
$$

and thus the collection $\left\{F_{n, l}^{\prime}: n \in \mathbb{N}, 0 \leq l \leq k\right\}$ is weakly relatively compact in $\mathbb{L}^{2}(\lambda)$. If we fix some point $x_{0} \in \bar{V}$, the set $\left\{F_{n, l}\left(x_{0}\right): n \in \mathbb{N}, 0 \leq k \leq n\right\}$ is also relatively compact in $\mathbb{R}$. 
From these observations it follows that we can find a subsequence $\left(n_{p}\right)_{p \in \mathbb{N}}$ and $k+1$ functions $\left(F_{\infty, l}\right)_{0 \leq l \leq k} \in \mathcal{F}(\bar{V}) \backslash\{0\}$ such that we are insured of the weak convergences

$$
\forall 0 \leq l \leq k, \quad \lim _{n \rightarrow \infty} F_{n, l}^{\prime}=F_{\infty, l}^{\prime}
$$

and of the uniform convergences

$$
\forall 0 \leq l \leq k, \quad \lim _{n \rightarrow \infty} F_{n, l}=F_{\infty, l}
$$

As a consequence, we get that

$$
\forall 0 \leq l \leq k, \quad\left\{\begin{array}{l}
\lim _{n \rightarrow \infty} \overline{\mathcal{E}}\left(F_{n, l}\right) \geq \overline{\mathcal{E}}\left(F_{\infty, l}\right) \\
\lim _{n \rightarrow \infty} \pi\left(F_{n, l}^{2}\right)=\pi\left(F_{\infty, l}^{2}\right)
\end{array}\right.
$$

which implies in fact that

$$
\max _{0 \leq l \leq k} \frac{\overline{\mathcal{E}}\left(F_{\infty, l}\right)}{\pi\left(F_{\infty, l}^{2}\right)} \leq \Lambda_{k}
$$

For any $0 \leq l \leq k$, let $A_{l}^{(k)}$ be a connected component of $\left\{F_{\infty, l}>0\right\}$ where the quotient $\overline{\mathcal{E}}\left(\mathbb{1}_{A_{l}^{(k)}} F_{\infty, l}\right) / \pi\left(\mathbb{1}_{A_{l}^{(k)}} F_{\infty, l}^{2}\right)$ is the smallest among all possible choices of such a component. Then we have that $\mathbb{1}_{A_{l}^{(k)}} F_{\infty, l} \in \mathcal{F}(\bar{V})$ and that

$$
\begin{aligned}
\bar{\lambda}_{0}\left(A_{l}^{(k)}\right) & \leq \frac{\overline{\mathcal{E}}\left(\mathbb{1}_{A_{l}^{(k)}} F_{\infty, l}\right)}{\pi\left(\mathbb{1}_{A_{l}^{(k)}} F_{\infty, l}^{2}\right)} \\
& \leq \frac{\overline{\mathcal{E}}\left(F_{\infty, l}\right)}{\pi\left(F_{\infty, l}^{2}\right)} \\
& \leq \Lambda_{k}
\end{aligned}
$$

We note that these sets $A_{l}^{(k)}, 0 \leq l \leq k$, are disjoint, because for any $0 \leq l \neq l^{\prime} \leq k, F_{\infty, l} F_{\infty, l^{\prime}}=$ $\lim _{n \rightarrow \infty} F_{n, l} F_{n, l^{\prime}}=0$, connected and open. Thus considering $A^{(k)}:=\left(A_{0}^{(k)}, \ldots, A_{k}^{(k)}\right) \in \mathcal{A}_{k}$, it appears that

$$
\Lambda\left(A^{(k)}\right) \leq \Lambda_{k}
$$

and then equality necessarily holds.

One can go further in this direction, but not much:

Remark 5 Let us recall the definition of Hausdorff topology. We denote by $d$ the natural distance on $\bar{V}$ and if $A$ is a subset of $\bar{V}$, then for $t \geq 0, A_{t}:=\{x \in \bar{V}: d(x, A) \leq t\}$ designates the $t$-enlargement of $A$. Next we consider the pseudo-metric $D$ which associates to nonempty subsets $A, B \subset \bar{V}$, the nonnegative number

$$
D(A, B):=\inf \left\{t \geq 0: A \subset B_{t}, B \subset A_{t}\right\}
$$

Even restricted to $\mathcal{A}_{0}, D$ is not yet a "true" metric because it does not separate different elements (except if the underlying graph is a tree): assume that $A \in \mathcal{A}_{0}$ contains a cycle, namely all the edge-segments $\left[x_{0}, x_{1}\right],\left[x_{1}, x_{2}\right], \ldots,\left[x_{p}, x_{0}\right]$, where $p \geq 2$ and the $x_{i}, 0 \leq i \leq 2$ are distinct elements of $V$. Then for any $z \in\left(x_{0}, x_{1}\right), A \backslash\{z\}$ still belongs to $\mathcal{A}_{0}$ and $D(A, A \backslash\{z\})=0$. Nevertheless, we can circumvent this drawback, by replacing $\mathcal{A}_{0}$ by $\widetilde{\mathcal{A}}_{0}$, the set of elements of $\mathcal{A}_{0}$ which are equal to the interior of their closure. This operation is harmless when trying to minimize $\bar{\lambda}_{0}$, because 
for $A \in \mathcal{A}_{0}$, we have $\bar{\lambda}_{0}\left(\widetilde{A}_{0}\right) \leq \bar{\lambda}_{0}\left(A_{0}\right)$, where $\widetilde{A}_{0}$ is the interior of the closure of $A_{0}$ (the inequality is even strict if $\widetilde{A}_{0} \neq A_{0}$, by Lemma 3 ). One can easily check that $D$ is a distance on $\widetilde{\mathcal{A}}_{0}$ and the corresponding topology is Hausdorff (except that traditionally one considers collections of compact subsets). The arguments of the proof of Lemma 4 can then be adapted to show that $\bar{\lambda}_{0}$ is lower semi-continuous on $\widetilde{\mathcal{A}}_{0}$. But this does not really help to deduce at once Lemma 4 from a more abstract principle, because it can be shown that the sublevel sets of $\bar{\lambda}_{0}$ (i.e. the sets of the form $\left\{A \in \widetilde{\mathcal{A}}_{0}: \bar{\lambda}_{0}(A) \leq t\right\}$ for some $t \geq 0$ ) are not compact as soon as the underlying graph is not a path (this is related to the fact we had to consider connected components at the end of the proof of Lemma 4, indeed one would have to relax the requirement of connectedness for the elements of $\widetilde{\mathcal{A}}_{0}$ ). It is more immediate to see that $\widetilde{\mathcal{A}}_{0}$ is not compact, since $\bar{\lambda}_{0}$ is not bounded there (consider elements of $\widetilde{\mathcal{A}}_{0}$ converging to a point of $V$ ). We also point out that $\bar{\lambda}_{0}$ is not continuous at any $A \in \widetilde{\mathcal{A}}_{0}$ which contains a cycle, as it can be observed by removing smaller and smaller closed intervals centered at a fixed virtual point of the cycle.

Despite the previous remark, it is possible to get some partial continuity results on $\bar{\lambda}_{0}$. Let $A \in \mathcal{A}_{0}$ be given, a point on its boundary is said to be good (and bad otherwise) if it also belongs to the boundary of the complementary set of $A$ (in particular boundary points of elements of $\widetilde{\mathcal{A}}_{0}$ are all good). If $x \in \partial A$ is good, we define for $t \in \mathbb{R}$,

$$
A_{x, t}:= \begin{cases}A \cup B(x, t) & , \text { if } t>0 \\ A & , \text { if } \mathrm{t}=0 \\ A \backslash \bar{B}(x,|t|) & , \text { if } t<0\end{cases}
$$

where $B(x, s)$ (respectively $\bar{B}(x, s)$ ) is the open (resp. closed) ball of radius $s>0$ centered at $x$. Then we have

Lemma 6 For good boundary points $x$ of $A \in \mathcal{A}_{0}$, the mapping

$$
\mathbb{R} \ni t \mapsto \bar{\lambda}_{0}\left(A_{x, t}\right)
$$

is continuous at 0 .

The following argument shows indeed that the above mapping is continuous in a neighbourhood of 0 .

\section{Proof}

We consider only the case where $x \in V$ and where $t$ goes to zero from above, the other situations are less embarrassing and are left to the reader. Coming back to the notations of Lemma 2, we define $W(t):=\widetilde{A}_{t, x} \backslash \widetilde{A}$. Let $r$ be the number of edge-segments $[x, y]$ such that $A^{\mathrm{c}} \cap[x, y]$ is a neighbourhood of $x$ in $[x, y]$. Then for $t>0$ small enough, $W(t)$ consists of exactly $r$ points escaping from $x$ in the direction of these particular edge-segments. To simplify notation, we write $\bar{\lambda}_{0}(t):=\bar{\lambda}_{0}\left(A_{x, t}\right), \widetilde{L}_{t}:=\widetilde{L}_{A_{x, t}}$ and $f_{t}:=f_{\widetilde{A}_{x, t}}$. Thus we have

$$
\sum_{y \in W(t)} \widetilde{L}_{t}(x, y) f_{t}(x)=\bar{\lambda}_{0}(t) f_{t}(x)+\sum_{y \in \widetilde{A}} \widetilde{L}_{A}(x, y)\left(f_{t}(y)-f_{t}(x)\right)
$$

and this shows that the l.h.s. is bounded for $t$ in a right neighbourhood of 0 (recall that $f_{t}$ takes its values in $[0,1]$ and that $\bar{\lambda}_{0}(t) \leq \bar{\lambda}_{0}(A)$ for $\left.t \geq 0\right)$. Since we have

$$
\lim _{t \rightarrow 0_{+}} \sum_{y \in W(t)} \widetilde{L}_{t}(x, y)=+\infty
$$

it follows that

$$
\lim _{t \rightarrow 0_{+}} f_{t}(x)=0
$$


This limit implies that if $\left(t_{n}\right)_{n \in \mathbb{N}}$ is a decreasing sequence converging to zero such that $\bar{\lambda}_{0}\left(t_{n}\right)$ and the restriction of $f_{t_{n}}$ to $\widetilde{A}$ converge, say respectively to $l$ and $f$, then we must have $\widetilde{L}_{A}[f]=-l f$ on $A \cap V$ and $f$ must vanish on $\partial A$. By a new invocation of Perron-Frobenius theorem, we get that $l=\bar{\lambda}_{0}(A)$ and $f=\widetilde{f}_{A}$, and through an usual compactness argument, that

$$
\lim _{t \rightarrow 0_{+}} \bar{\lambda}_{0}(t)=\bar{\lambda}_{0}(A)
$$

We can now give a first result about a minimizing pseudo-partition as in Lemma 4, which will be important for the construction of eigenvectors of birth and death processes. It also serves as a justification of the introduction of the continuous space $\bar{V}$.

Proposition 7 For $0 \leq k \leq N$, let $A^{(k)}:=\left(A_{0}^{(k)}, \ldots, A_{k}^{(k)}\right) \in \mathcal{A}_{k}$ be such that $\Lambda_{k}=\Lambda\left(A^{(k)}\right)$. Assume that the intersection of the boundaries of any three distinct domains of this pseudo-partition is empty. Then we have

$$
\forall 0 \leq l \leq k, \quad \bar{\lambda}_{0}\left(A_{l}^{(k)}\right)=\Lambda_{k}
$$

For instance this is always true if $k=1$. Another way to insure the above intersection property is to check that for $0 \leq l \leq k$, we have $\partial A_{l}^{(k)} \cap V_{3}=\emptyset$, where $V_{3}$ stands for the set of vertices of the graph $(V, E)$ whose degree is larger or equal to 3. In particular, this is satisfied when $(V, E)$ is either a path or a cycle.

\section{Proof}

So let $A^{(k)}$ satisfy the above hypotheses. We define $I$ as the collection of indices $0 \leq l \leq k$ such that $\bar{\lambda}_{0}\left(A_{l}^{(k)}\right)=\Lambda\left(A^{(k)}\right)$ and let us assume that $I \neq\{0,1, \ldots, k\}$. If $I$ is reduced to a singleton $\{i\}$, then a contradiction follows easily: let $x \in \partial A_{i}^{(k)}$, two cases are possible. Either $x$ does not belong to any of the other boundaries $\partial A_{l}^{(k)}$, for $0 \leq l \neq i \leq k$, and we can extend a little $A_{i}^{(k)}$ by moving $x$ toward the exterior, if $x$ is good, or just add $x$ to $A_{i}^{(k)}$ if $x$ is bad. As a result, $\Lambda\left(A^{(k)}\right)=\bar{\lambda}_{0}\left(A_{i}^{(k)}\right)$ will decrease (either continuously if $x$ is good or by a jump downward if $x$ is bad), in contradiction with the fact that $A^{(k)}$ is a minimizer. If $x$ was to belong to another boundary, say to $\partial A_{j}^{(k)}$, with $0 \leq j \neq i \leq k$, then $x$ is good and $j$ is necessary unique by our assumption. Thus we can still extend a little $A_{i}^{(k)}$ by moving the boundary $x$ toward the exterior (maybe in several directions as in the proof of Lemma 6 if $x$ was to belong to $V$ ), this will also reduce a little $A_{j}^{(k)}$, but since we started with $\bar{\lambda}_{0}\left(A_{i}^{(k)}\right)>\bar{\lambda}_{0}\left(A_{j}^{(k)}\right)$, by Lemma 6 the initial tendency will again be that $\Lambda\left(A^{(k)}\right)$ has to decrease. If $I$ is not a singleton, we can reduce it by changing a little $A^{(k)}$ in the following way (keeping it as a minimizer). By irreducibility, there exist an index $i \in I$ such that one of the good boundary point $x$ of $\partial A_{i}^{(k)}$ does not belong to the boundaries $\partial A_{l}^{(k)}$, for $l \in I \backslash\{i\}$, by our main assumption. Then as above, we can push outward a little $x$ so that $i$ get out of $I$, without changing the relation $\Lambda_{k}=\Lambda\left(A^{(k)}\right)$ and keeping satisfied our assumption about three by three intersections. Repeating this operation, we end up with $I$ equal to a singleton and to a contradiction as before. Thus we must have $I=\{0,1, \ldots, k\}$, which is the first announced result. The end of the proposition is immediate.

Let us emphatize that the condition on three by three empty intersections is not just technical.

Example 8 Consider the following generator with $N=3$

$$
L=\left(\begin{array}{cccc}
-10 & 4 & 4 & 2 \\
4 & -4 & 0 & 0 \\
4 & 0 & -4 & 0 \\
2 & 0 & 0 & -2
\end{array}\right)
$$


The corresponding graph is a star, with 0 at the center, 1 and 2 are a distance 1 of 0 and 3 is at distance 2 of 0 . Then for $k=2$, we have $\Lambda_{2}=4$ and a minimizing pseudo-partition is given by $([1,0),[2,0),[3,0))$ but we have $\bar{\lambda}_{0}([1,0))=4=\bar{\lambda}_{0}([2,0))<\bar{\lambda}_{0}([3,0))=2$. Nevertheless we can find a minimizing pseudo-partition whose elements have the same $\bar{\lambda}_{0}$, it suffices to consider $([1,0),[2,0),[3, x))$ where $x$ is the middle of $[3,0]$. This is a general fact, as it is stated below.

Let say that $A=\left(A_{0}, \ldots, A_{k}\right) \in \mathcal{A}_{k}$ is isospectral or well-balanced if we have $\lambda_{0}\left(A_{l}\right)=\Lambda(A)$ for any $0 \leq l \leq k$.

Proposition 9 Let $0 \leq k \leq N$ be given, there always exists a minimizing $A \in \mathcal{A}_{k}$ for $\Lambda$ which is well-balanced.

\section{Proof}

Since it will not be useful for us in this paper, we will only sketch the proof of the existence of well-balanced pseudo-partitions. The idea is that given $A \in \mathcal{A}_{0}$, we can find a decreasing family $\left(A_{t}\right)_{0 \leq t<1}$ with $A_{0}=A$ and such that the mapping $[0,1) \ni t \mapsto \bar{\lambda}_{0}\left(A_{t}\right)$ is continuous and such that $\lim _{t \rightarrow 1-}-\bar{\lambda}_{0}\left(A_{t}\right)=+\infty$. Indeed, we choose $x \in \partial A$ and $y \in A \cap V$ such that $[x, y]$ is included into an edge-segment. We begin by replacing the part $[y, x)$ of $A$ by $[y, z)$ with $z$ going from $x$ to $y$. Using the arguments of the proof of Lemma 6 , this can be done continuously for $\bar{\lambda}_{0}$. If $A \cap V$ was reduced to $y$, then $\bar{\lambda}_{0}$ goes to $+\infty$ when $z$ goes to $y$. Otherwise, when $z$ attains $y$, two situations are possible. Either the set $B$ obtained by removing $[y, x)$ from $A$ is no longer connected, then we replace it by the connected component with the smaller $\bar{\lambda}_{0}$, this does not induce a jump for $\bar{\lambda}_{0}$, because if we decompose a set $S$ into its connected components, say the $\left(S_{i}\right)_{1 \leq i \leq r}$, then we have $\bar{\lambda}_{0}(S)=\min _{1 \leq i \leq r} \bar{\lambda}_{0}\left(S_{i}\right)$. Otherwise, if $B$ is connected, we just keep it. In both cases, $y$ becomes a boundary point of the current set and we can iterate the previous procedure.

Next let a minimizing $A=\left(A_{0}, \ldots, A_{k}\right) \in \mathcal{A}_{k}$ for $\Lambda$ be given. If $0 \leq l \leq k$ is such that $\bar{\lambda}_{0}\left(A_{l}\right)<\Lambda(A)$, using the above property we can reduce $A_{l}$ until its $\bar{\lambda}_{0}$ increase to $\Lambda(A)$. Doing so for all such indices $0 \leq l \leq k$, we end up with a well-balanced pseudo-partition still minimizing $\Lambda$.

There is another interesting kind of pseudo-partitions: for $0 \leq k \leq N$, call $A \in \mathcal{A}_{k}$ a quasi-partition if $\bar{V}$ is covered by the closure of the union of the $A_{l}$, for $0 \leq l \leq k$. Contrary to well-balanced pseudo-partitions, such a pseudo-partition may not exist, for instance consider Example 8 with $k=1$. Up to a change of order, there is a unique minimizer for $\Lambda_{1}$, which is $([1,0),[2,0))$ and if one try to extend one of its elements, one has to diminish the other to keep satisfied the openness requirement. Nevertheless, there is a simple criterion insuring that a pseudo-partition is a quasipartition.

Proposition 10 For $0 \leq k \leq N$, let $A^{(k)}:=\left(A_{0}^{(k)}, \ldots, A_{k}^{(k)}\right) \in \mathcal{A}_{k}$ be such that $\Lambda_{k}=\Lambda\left(A^{(k)}\right)$ and verifying that for $0 \leq l \leq k$, we have $\partial A_{l}^{(k)} \cap V_{3}=\emptyset$. Then $A^{(k)}$ is a quasi-partition. In particular when the graph $(V, E)$ is a path or a cycle, a minimizing pseudo-partition is always a well-balanced quasi-partition.

\section{Proof}

By Proposition 7, we already know that $A^{(k)}$ is well-balanced. But if $\bar{V}$ was not covered by the closure of the union of the $A_{l}^{(k)}$, for $0 \leq l \leq k$, then one could extend a little one of them, without disturbing the others elements, because of our assumption. By this procedure we would get a new minimizing partition (if $k \geq 1$, otherwise the result is trivial anyway), which is no longer well-balanced. But if the extension is small enough, the assumption is also preserved and we get a contradiction with Proposition 7. 
Another result we will need to obtain the shape of the eigenvectors associated to birth and death processes is that for $A \in \mathcal{A}_{0}$, the "landscape" of $F_{A}$ is that of hills without lake.

Proposition 11 Let $A \in \mathcal{A}_{0}$ and $x \in A \cap V$ be given and assume that $\mathcal{N}(x)$, the set of neighbours of $x$ in the graph $(V, E)$, is included into the closure of $A$. Then we have

$$
\min _{y \in N(x)} F_{A}(y) \leq F_{A}(x)
$$

and the inequality is strict if $A \neq \bar{V}$.

\section{Proof}

Let $f_{A}$ designate the restriction of $F_{A}$ to $V$. Then for $x$ as above, the relation given in Lemma 2 can be rewritten as

$$
L\left[f_{A}\right](x)=-\bar{\lambda}_{0}(A) f_{A}(x)
$$

So if we had $\min _{y \in N(x)} f_{A}(y)>f_{A}(x)$, the l.h.s. would be positive, while the r.h.s. is nonnegative, a contradiction. Indeed, if $A \neq \bar{V}$, we have $\bar{\lambda}(A)>0$ (because $\overline{\mathcal{E}}\left(F_{A}\right)$ cannot be null) and the r.h.s. is positive and thus it must exist some $y \in \mathcal{N}(x)$ satisfying $F_{A}(y)<F_{A}(x)$.

To finish this section, even it will not be useful for this paper, let us mention a general comparison between the eigenvalues and the quantities introduced above.

Proposition 12 For any $0 \leq k \leq N$, we have

$$
\lambda_{k} \leq \Lambda_{k}
$$

\section{Proof}

It is based on the variational principle asserting that

$$
\lambda_{k}=\min _{H: \operatorname{dim}(H)=k+1} \max _{f \in H \backslash\{0\}} \frac{\mathcal{E}(f)}{\pi\left(f^{2}\right)}
$$

where the minimum is over all subspace of $\mathcal{F}(V)$ of dimension $k+1$. So let $A^{(k)}:=\left(A_{0}^{(k)}, \ldots, A_{k}^{(k)}\right) \in$ $\mathcal{A}_{k}$ be as in Lemma 4 , consider for $0 \leq l \leq k, F_{k, l}:=F_{A_{l}^{(k)}}$ and denote by $f_{k, l}$ its restriction to $V$. Next let $H$ the subspace of $\mathcal{F}(V)$ generated by the functions $f_{k, l}$, for $0 \leq, l \leq k$, which are clearly linearly independent. For any $h \in H$, there exist coefficients $a_{0}, \ldots, a_{k} \in \mathbb{R}$, such that $h=\sum_{0 \leq l \leq k} a_{l} f_{k, l}$, which is also the restriction to $V$ of the function $F=\sum_{0 \leq l \leq k} a_{l} F_{k, l}$. Thus it appears that

$$
\begin{aligned}
\mathcal{E}(h) & \leq \overline{\mathcal{E}}(F) \\
& =\sum_{0 \leq l \leq k} a_{l}^{2} \mathcal{E}\left(F_{k, l}\right)
\end{aligned}
$$

On the other hand, we have

$$
\begin{aligned}
\pi\left(h^{2}\right) & =\sum_{0 \leq l \leq k} a_{l}^{2} \pi\left(f_{k, l}^{2}\right) \\
& =\sum_{0 \leq l \leq k} a_{l}^{2} \pi\left(F_{k, l}^{2}\right)
\end{aligned}
$$


It follows that

$$
\begin{aligned}
\frac{\mathcal{E}(h)}{\pi\left(h^{2}\right)} & \leq \max _{0 \leq l \leq k} \frac{\mathcal{E}\left(F_{k, l}\right)}{\pi\left(F_{k, l}^{2}\right)} \\
& =\Lambda_{k}
\end{aligned}
$$

and by consequence

$$
\begin{aligned}
\lambda_{k} & \leq \max _{f \in H \backslash\{0\}} \frac{\mathcal{E}(f)}{\pi\left(f^{2}\right)} \\
& \leq \Lambda_{k}
\end{aligned}
$$

Conversely, assume that for some $0 \leq k \leq N$, there exists an eigenfunction $\varphi$ associated to $\lambda_{k}$ which has $k+1$ nodal domains. Let $\bar{\varphi}$ be the extension of $\varphi$ on $\bar{V}$ which is affine on any edge-segment. Let $A$ be a nodal domain of $\bar{\varphi}$, resorting to Perron-Frobenius theorem as in the proof of Lemma 2 , one shows that the restriction of $\bar{\varphi}$ to $A$ (extended by zero outside) is proportional to $F_{A}$, so that $\lambda_{0}(A)=\lambda_{k}$ (see also section 2 of Friedman [11]). Thus by considering the pseudo-partition made of the nodal domains of $\bar{\varphi}$, we get that $\Lambda_{k} \leq \lambda_{k}$ and thus $\Lambda_{k}=\lambda_{k}$. In particular, we always have $\Lambda_{1}=\lambda_{1}$. In next sections, we will directly prove that the equality $\Lambda_{k}=\lambda_{k}$ holds for any $0 \leq k \leq N$, if the graph $(V, E)$ is a tree. Alternatively, one can use a result of Biyıkoğlu [2] saying that generically, the previous nodal property is satisfied by a generator whose associated graph is a tree (if it is a path, a stronger property is true, since all eigenfunctions associated to $\lambda_{k}$ have $k+1$ nodal domains by the result of Gantmacher and Krein [13] recalled at the beginning of the introduction). Nevertheless, the equality $\Lambda_{k}=\lambda_{k}$ cannot be general, for instance it is not verified with $k=2$ for the generator corresponding to the simple random walk on the cycle $\mathbb{Z} /(N+1) \mathbb{Z}$, as soon as $N \geq 1$. But we believe the following is true

Conjecture 13 There exists an universal constant $\chi>1$ such that for any finite irreducible and reversible generator on $V$, we have

$$
\forall 0 \leq k \leq N, \quad \Lambda_{k} \leq \chi \lambda_{k}
$$

An equally interesting conjecture is to allow $\chi$ to depend on $k \in \mathbb{N}$. But one reason which induced us to believe in the stronger version of Conjecture 13, is that it can be shown that it is satisfied by generator of the form $L=\pi-$ Id (namely, for any $x, y \in V, L(x, y)=\pi(y)-\delta_{x=y}$ ), with $\chi=2$. Our concern about this question comes from higher order Cheeger inequalities, as we explain it now.

If $A \subset V$ is nonempty, we associate to it the quantity

$$
\iota(A):=\frac{\pi\left(\mathbb{1}_{A^{\mathrm{c}}} L\left[\mathbb{1}_{A}\right]\right)}{\pi(A)}
$$

and we introduce the $k^{\text {th }}$-order isoperimetric constant as

$$
I_{k}:=\max _{\left(A_{0}, \ldots, A_{k}\right) \in \mathcal{A}_{k}(V)} \min _{0 \leq l \leq k} \iota\left(A_{l}\right)
$$

(where $\mathcal{A}_{k}(V)$ is defined as $\mathcal{A}_{k}$, but on $V$ instead of $\bar{V}$ ). Then Conjecture 13 would imply that there exists an universal constant $\chi^{\prime}>0$ such that

$$
\forall 0 \leq k \leq N, \quad \chi^{\prime} \frac{I_{k}^{2}}{|L|} \leq \lambda_{k} \leq 2 I_{k}
$$

where $|L|:=\max _{x \in V}|L(x, x)|$. The case $k=1$ is well-known and corresponds to the traditional discrete Cheeger inequality (with $\chi^{\prime}=1 / 2$, see Lawler and Sokal [16]). It was first obtained on compact Riemannian manifolds by Cheeger [3]. But it would not be very difficult, through appropriate approximations, to extend (4) back to this continuous setting. 


\section{Construction and shape of eigenvectors}

Here we return to the situation of birth and death processes. Using results of previous section, we provide a direct construction of the corresponding eigenvectors. The description given in the introduction will follow.

So let $L$ be a generator given on $V$ as in (1). We fix $1 \leq k \leq N$ and we consider a corresponding well-balanced quasi-partition $A^{(k)}:=\left(A_{0}^{(k)}, \ldots, A_{k}^{(k)}\right) \in \mathcal{A}_{k}$ which is minimizing for $\Lambda$, namely $\Lambda\left(A^{(k)}\right)=\Lambda_{k}$. Without loss of generality, we assume that this quasi-partition is naturally ordered: for $0 \leq i<j \leq N$, all elements of $A_{i}^{(k)}$ are smaller than all elements of $A_{j}^{(k)}$. For all $0 \leq l \leq k \leq N$, let $f_{k, l}$ designate the restriction of the minimizer $F_{A_{l}^{(k)}}$ to $A_{k, l}$, which will denote $A_{l}^{(k)} \cap V$. Next, to any $k$-tuple $\left(r_{k, 1}, \ldots, r_{k, k}\right) \in \mathbb{R}^{k}$, we associate the function $\psi_{k} \in \mathcal{F}(V)$ defined by

$$
\forall x \in V, \quad \psi_{k}(x):= \begin{cases}f_{k, 0}(x) & , \text { if } x \in A_{k, 0} \\ r_{k, l} f_{k, l}(x) & , \text { if } x \in A_{k, l}, \text { for some } 1 \leq l \leq N \\ 0 & , \text { otherwise }\end{cases}
$$

Then we have

Theorem 14 There exists a unique choice of $\left(r_{k, 1}, \ldots, r_{k, k}\right) \in \mathbb{R}^{k}$ such that $L\left[\psi_{k}\right]=-\Lambda_{k} \psi_{k}$.

\section{Proof}

Since $A^{(k)}$ is well-balanced, Lemma 2 shows that

$$
\forall 0 \leq l \leq N, \forall x \in A_{k, l}, \quad \widetilde{L}_{A_{l}^{(k)}}\left[\psi_{k}\right](x)=-\Lambda_{k} \psi_{k}(x)
$$

where $\psi_{k}$ has been extended by zero at the virtual points of the boundaries of the elements of the quasi partition (note that on boundary points belonging to $V, \psi_{k}$ already vanishes by definition), and this is true independently of the choice of $\left(r_{k, 1}, \ldots, r_{k, k}\right) \in \mathbb{R}^{k}$. In particular, if a point $x \in V$ and its nearest neighbour(s) belong to $A_{k, l}$ for some $0 \leq l \leq k$, then we get $L\left[\psi_{k}\right](x)=-\Lambda_{k} \psi_{k}(x)$. We will now see how to choose successively $r_{k, 1}, r_{k, 2}, \ldots$ up to $r_{k, k}$ so that the latter relation is true on $V$.

Let $a_{k, 0}^{+}$be the largest point of $A_{k, 0}$ and $a_{k, 1}^{-}$be the smallest point of $A_{k, 1}$. We first consider the case where $a_{k, 1}^{-}=a_{k, 0}^{+}+1$. We want to find $r_{k, 1} \in \mathbb{R}$ such that

$$
\begin{aligned}
& \widetilde{L}_{A_{0}^{(k)}}\left[\psi_{k}\right]\left(a_{k, 0}^{+}\right)=L\left[\psi_{k}\right]\left(a_{k, 0}^{+}\right) \\
& \widetilde{L}_{A_{1}^{(k)}}\left[\psi_{k}\right]\left(a_{k, 1}^{-}\right)=L\left[\psi_{k}\right]\left(a_{k, 1}^{-}\right)
\end{aligned}
$$

Indeed, the first equation is asking for

$$
\widetilde{L}_{A_{0}^{(k)}}\left(a_{k, 0}^{+}, c_{k, 0}\right)\left(0-f_{k, 0}\left(a_{k, 0}^{+}\right)\right)=L\left(a_{k, 0}^{+}, a_{k, 1}^{-}\right)\left(r_{k, 1} f_{k, 1}\left(a_{k, 1}^{-}\right)-f_{k, 0}\left(a_{k, 0}^{+}\right)\right)
$$

where $c_{k, 0}$ is the virtual boundary point of $A_{0}^{(k)}$, which is also the left hand side boundary point of $A_{1}^{(k)}$, since $A^{(k)}$ is a quasi-partition. This gives

$$
r_{k, 1}=\frac{f_{k, 0}\left(a_{k, 0}^{+}\right)}{f_{k, 1}\left(a_{k, 1}^{-}\right)}\left(1-\frac{\widetilde{L}_{A_{0}^{(k)}}\left(a_{k, 0}^{+}, c_{k, 0}\right)}{L\left(a_{k, 0}^{+}, a_{k, 1}^{-}\right)}\right)
$$

In a similar fashion, the second equation leads to

$$
r_{k, 1}=\frac{f_{k, 0}\left(a_{k, 0}^{+}\right)}{f_{k, 1}\left(a_{k, 1}^{-}\right)}\left(\frac{L\left(a_{k, 1}^{-}, a_{k, 0}^{+}\right)}{L\left(a_{k, 1}^{-}, a_{k, 0}^{+}\right)-\widetilde{L}_{A_{1}^{(k)}}\left(a_{k, 1}^{-}, c_{k, 0}\right)}\right)
$$


This is indeed the same solution as before, because

$$
\frac{1}{\pi\left(a_{k, 0}^{+}\right) L\left(a_{k, 0}^{+}, a_{k, 1}^{-}\right)}=\frac{1}{\pi\left(a_{k, 0}^{+}\right) L_{A_{0}^{(k)}}\left(a_{k, 0}^{+}, c_{k, 0}\right)}+\frac{1}{\pi\left(a_{k, 1}^{-}\right) L_{A_{1}^{(k)}}\left(a_{k, 1}^{-}, c_{k, 0}\right)}
$$

which comes from the fact that in $\bar{V}$, the length from $a_{k, 0}^{+}$to $a_{k, 1}^{-}$is the length from $a_{k, 0}^{+}$to $c_{k, 0}$ added to the length from $c_{k, 0}$ to $a_{k, 1}^{-}$. Then using that $\pi\left(a_{k, 1}^{-}\right)=\pi\left(a_{k, 0}^{+}\right) L\left(a_{k, 0}^{+}, a_{k, 1}^{-}\right) / L\left(a_{k, 1}^{-}, a_{k, 0}^{+}\right)$, one get the equality of the above expressions for $r_{k, 1}$.

To have a better picture of what we have just done, let us isometrically embed $\bar{V}$ into $\mathbb{R}_{+}$by $\iota$. Furthermore requiring that $\iota(0)=0$, we get in particular that

$$
\forall 1 \leq k \leq N, \quad \iota(k)=\sum_{0 \leq l<k} \frac{1}{\pi(l) L(l, l+1)}
$$

Then, multiplying (7) by $\pi\left(a_{k, 0}^{+}\right)$, interpreting the inverse of the quantities $\pi\left(a_{k, 0}^{+}\right) \widetilde{L}_{A_{0}^{(k)}}\left(a_{k, 0}^{+}, c_{k, 0}\right)$ and $\pi\left(a_{k, 0}^{+}\right) L\left(a_{k, 0}^{+}, a_{k, 1}^{-}\right)$as the distances $\left|\iota\left(a_{k, 0}^{+}\right)-\iota\left(c_{k, 0}\right)\right|$ and $\left|\iota\left(a_{k, 0}^{+}\right)-\iota\left(a_{k, 1}^{-}\right)\right|$and resorting to Thales' theorem, it appears that the above equations are just asking for the three points

$$
\left(\iota\left(a_{k, 0}^{+}\right), f_{k, 0}\left(a_{k, 0}^{+}\right)\right) \quad\left(\iota\left(c_{k, 0}\right), 0\right) \quad\left(\iota\left(a_{k, 1}^{-}\right), r_{k, 1} f_{k, 1}\left(a_{k, 1}^{-}\right)\right)
$$

to be on a same line in $\mathbb{R}^{2}$ (in particular $r_{k, 1}$ has to be negative).

We now come to the situation where $a_{k, 1}^{-}=a_{k, 0}^{+}+2$, which means that the point $c_{k, 0}$ at the intersection of $\partial A_{0}^{(k)}$ and $\partial A_{1}^{(k)}$ belongs to $V$. Then the conditions (5) and (6) are already satisfied, independently of $r_{k, 1}$, but what we want is that

$$
L\left[\psi_{k}\right]\left(c_{k, 0}\right)=0
$$

since $\psi_{k}\left(c_{k, 0}\right)=0$. But again this amounts to asking for the three points (8) to be on a same line, which leads to a unique choice of $r_{k, 1}$.

To summarize, by the above adjustment of $r_{k, 1}$, the relation $L\left[\psi_{k}\right]=-\Lambda_{k} \psi_{k}$ is true on $\left(A_{0}^{(k)} \sqcup\right.$ $\left.\left\{c_{0, k}\right\} \sqcup A_{1}^{(k)}\right) \cap V$, except on its right most point $a_{k, 1}^{+}$(if the right hand side boundary of $A_{1}^{(k)}$ does not belong to $V$ ). But the above procedure can be iterated to choose $r_{k, 2}, r_{k, 3}, \ldots$ up to $r_{k, k}$, to extend the relation $L\left[\psi_{k}\right]=-\Lambda_{k} \psi_{k}$ on the whole state space $V$. For instance, with obvious notations, the choice of $r_{k, 2}$ is such that the three points

$$
\left(\iota\left(a_{k, 1}^{+}\right), r_{k, 1} f_{k, 1}\left(a_{k, 1}^{+}\right)\right) \quad\left(\iota\left(c_{k, 1}\right), 0\right) \quad\left(\iota\left(a_{k, 2}^{-}\right), r_{k, 2} f_{k, 2}\left(a_{k, 2}^{-}\right)\right)
$$

are on a same line in $\mathbb{R}^{2}$.

To be convinced we get all the eigenvalues of $-L$ in this way, it is sufficient to check that all the $\Lambda_{k}$, for $0 \leq k \leq N$, are distinct:

Lemma 15 The finite sequence $\left(\Lambda_{k}\right)_{0 \leq k \leq N}$ is increasing.

\section{Proof}

We have already seen in Section 2 that in general the finite sequence $\left(\Lambda_{k}\right)_{0 \leq k \leq N}$ is nondecreasing. Assume now that there exists $0 \leq k<N$ such that $\Lambda_{k}=\Lambda_{k+1}$ and let $A^{(k)}:=\left(A_{0}^{(k)}, \ldots, A_{k}^{(k)}\right)$ and $A^{(k+1)}:=\left(A_{0}^{(k+1)}, \ldots, A_{k+1}^{(k+1)}\right)$ be corresponding minimizing ordered quasi-partitions.

As in the proof of Theorem 14, we identify isometrically $\bar{V}$ and $[0, \iota(N)]$ through $\iota$. Then Lemma 3 and Lemma 6 enable to see that the mapping

$$
(0, \iota(N)) \ni t \quad \mapsto \quad \bar{\lambda}_{0}([0, t))
$$


is continuously decreasing. Thus there is a unique $0<c<\iota(N)$ such that $\bar{\lambda}_{0}([0, c))=\Lambda_{k}=\Lambda_{k+1}$, which means that $A_{0}^{(k)}=[0, c)=A_{0}^{(k+1)}$. Next considering the mapping

$$
(\iota(c), \iota(N)) \ni t \mapsto \bar{\lambda}_{0}([\iota(c), t))
$$

we deduce that $A_{1}^{(k)}=A_{1}^{(k+1)}$ and iteratively, we get that for any $0 \leq l \leq k, A_{l}^{(k)}=A_{l}^{(k+1)}$. It would follow that $A_{k+1}^{(k+1)}=\emptyset$, but this is forbidden, so $\Lambda_{k}<\Lambda_{k+1}$.

The same proof shows that for birth and death processes, the ordered pseudo-partition $A^{(k)} \in \mathcal{A}_{k}$ minimizing $\Lambda$ is unique, for any $0 \leq k \leq N$, since we have already seen that it is necessarily a quasi-partition. So from now on, the meaning of $A^{(k)}, A_{l}^{(k)}$ and $A_{k, l}$ will no longer be ambiguous and as in the introduction, we will write $A_{k, l}:=\llbracket a_{k, l}^{-}, a_{k, l}^{+} \rrbracket$.

By Lemma 15, we must have

$$
\forall 0 \leq k \leq N, \quad \Lambda_{k}=\lambda_{k}
$$

and even

$$
\forall 0 \leq k \leq N, \quad \psi_{k}=\varphi_{k}
$$

because of our conventions of normalization and next result.

Lemma 16 For any $1 \leq k \leq N, f_{k, 0}$ is decreasing on $A_{k, 0}$, so $f_{k, 0}(0)=\max _{A_{k, 0}} f_{k, 0}=1$.

\section{Proof}

If $A_{k, 0}$ is reduced to a singleton, there is nothing to prove. Otherwise, by Proposition 11 applied to $x=0$, we have $f(0)>f(1)$. Furthermore, this proposition also shows that it cannot exist $x-1$, $x$ and $x+1$, all three of them in $A_{k, 0}$, such that $f_{k, 0}(x-1)>f_{k, 0}(x) \leq f_{k, 0}(x+1)$. It is then easy to deduce that $f_{k, 0}$ has to be decreasing on $A_{k, 0}$.

Similar arguments based on Proposition 11 show that $f_{k, k}$ is increasing on $A_{k, k}$ and that for $0<l<k$, there exist two successive or equal point(s) $e_{k, l}^{-}, e_{k, l}^{+}$in $A_{k, l}$, so that, $f_{k, k}$ is increasing on $\llbracket a_{k, l}^{-}, e_{k, l}^{-} \rrbracket$ and decreasing on $\llbracket e_{k, l}^{+}, e_{k, l}^{+} \rrbracket$ and $f_{k, l}\left(e_{k, l}^{-}\right)=f_{k, l}\left(e_{k, l}^{+}\right)$. This implies the monotonicity results announced in the introduction, since one would have noticed that the parameters $\left(r_{k, 1}, \ldots, r_{k, k}\right) \in \mathbb{R}^{k}$ appearing in Theorem 14 have to be alternated: $r_{k, l}$ is negative for odd $1 \leq l \leq k$ and positive otherwise.

We now come to the interlacing property presented in the introduction. By the above considerations, we have that for $0<k \leq N$, the finite sequence $\left(c_{k, l}\right)_{0 \leq l<k}$ corresponds to the boundary points of the elements of $A^{(k)}$. By the pigeonhole principle, if the interlacing property was not true, we could find $0<k<N$ and $-1 \leq l<k$ such that $\left(c_{k, l}, c_{k, l+1}\right)$ does not contain any point from $\left(c_{k+1, l}\right)_{0 \leq l<k+1}$ (with the convention that $c_{k,-1}=0$ and $\left.c_{k, k}=N\right)$. But this would mean that $A_{l}^{(k)}$ is included into some set $A_{l^{\prime}}^{(k+1)}$, with $0 \leq l^{\prime} \leq k+1$, and thus that $\Lambda_{k+1}=\bar{\lambda}_{0}\left(A_{l^{\prime}}^{(k+1)}\right) \leq \bar{\lambda}_{0}\left(A_{l}^{(k)}\right)=\Lambda_{k}$, which is not possible.

To finish this section, let us mention that the presented construction can be translated into an algorithm to compute the eigendecomposition of $L$.

Remark 17 We begin by noticing that given $A \in \mathcal{A}_{0}$, it is not difficult to approximate $\bar{\lambda}_{0}(A)$ and $f_{\widetilde{A}}$. For instance, this can be done by iterating the sub-Markovian operator Id $-\widehat{L}_{A} /\left|\widehat{L}_{A}\right|$ on $A \cap V$, where $\widehat{L}_{A}$ was introduced in the proof of Lemma 2 and where we recall that $\left|\widehat{L}_{A}\right|:=$ 
$\max _{x \in A \cap V}\left|\widehat{L}_{A}(x, x)\right|$. But there exist other ways to do it, especially in higher dimension or in continuous setting, see for instance [8] for an interacting particle approach. Note also that in the linear setting of birth and death chains, once $\bar{\lambda}_{0}(A)$ is known, it is very easy to deduce $f_{\widetilde{A}}$, recursively by letting $x$ going from the left to the right of the discrete interval $A \cap V$ in the relation of Lemma 2. Let write $A=\left(c_{-}, c_{+}\right)$and $A \cap V=\llbracket a_{-}, a_{+} \rrbracket$. Taking into account the minimizing feature of $F_{A}$, one can show that

$$
\partial_{c_{+}} \bar{\lambda}_{0}(A)=-\frac{\pi^{2}\left(a_{+}\right) \widetilde{L}_{A}^{2}\left(a_{+}, c_{+}\right) f_{\widetilde{A}}^{2}\left(a_{+}\right)}{\pi\left(f_{\widetilde{A}}^{2}\right)}
$$

at least if $c_{+}$is virtual (and where $\partial_{c_{+}}$is the differentiation with respect to $c_{+}$in the natural length structure of $\bar{V}$ ). See also Theorem 2.6 of Friedman [11].

These are the necessary ingredients to apply usual optimization algorithms to find the global minima of $\Lambda$ over the subset of $\mathcal{A}_{k}$ consisting of ordered quasi-partitions, for a given $1 \leq k \leq N$. One can also look for the global minima of the functional $H$ defined on ordered quasi-partitions $A=\left(A_{0}, \ldots, A_{k}\right)$ by

$$
H(A):=\sum_{0 \leq l<k}\left(\bar{\lambda}_{0}\left(A_{l}\right)-\bar{\lambda}_{0}\left(A_{l+1}\right)\right)^{2}
$$

since we have seen that it is attained at $A^{(k)}$. But this property is not true for more general graphs than paths.

For practical implementation, it is certainly convenient to represent a quasi-partition by its boundary points. If one has already computed $\lambda_{k}$ and $\varphi_{k}$, the interlacing property can be used to a priori initialize the algorithm approximating $\lambda_{k+1}$ and $\varphi_{k+1}$.

It could be interesting to understand how such kind of algorithms work on more general graphs, even if a minimizing pseudo-partition for $\Lambda_{k}$ is no longer directly linked with $\varphi_{k}$ (for instance, the $\Lambda_{k}$ with $k$ even are not very relevant for cycles). But if Conjecture 13 was to be true, $\Lambda_{k}$ could serve as an estimator for $\lambda_{k}$, up to the universal factor $\chi$.

\section{Generical properties}

To end the proof of the results announced in the introduction, we will consider here generical properties of eigenvectors associated to birth and death processes. But we will also be interested in the more case of generators whose underlying graph is a tree, because the previous constructions can be generically extended to them. As a consequence, we will see that the identity $\Lambda_{k}=\lambda_{k}$ always holds for them, for any $0 \leq k \leq N$.

So here the generators $L$ will be random, in a birth and death process setting, to begin with. There are several ways to device distributions on them, for instance that we alluded to in the introduction, by sampling all the rates independently according to laws absolutely continuous with respect to the Lebesgue measure on $(0,+\infty)$. But from a technical point of view, this is not very convenient, it is better first to fix the reversible probability $\pi$ and next to sample independently the birth rates (the death rates being then imposed by $\pi$ ). Indeed, one is even getting more general results in this way (as long as a.s. behaviors are concerned, via Fubini's theorem), because it is easy to check that if all the rates are choosen independently, then conditionally on the reversible probability $\pi$ (which is now random, but it only depends on the quantities $\left(b_{x} / d_{x+1}\right)_{0 \leq x<N}$ ), the birth rates are independent. Furthermore, all their laws are absolutely continuous with respect to 
the Lebesgue measure on $(0,+\infty)$, if that property was verified by the laws of the rates (which were not assumed to be the same for all the rates). Thus from now on, we will assume that

$$
\left\{\begin{array}{l}
\text { the birth rates }\left(b_{x}\right)_{0 \leq x<N} \text { are independent and their laws are } \\
\text { absolutely continuous with respect to the Lebesgue measure on }(0,+\infty)
\end{array}\right.
$$

The reversible probability $\pi$ is fixed (and positive on $V$ ), so that the death rates are given by

$$
\forall 0<x \leq N, \quad d_{x}=\frac{\pi(x-1)}{\pi(x)} b_{x-1}
$$

In fact the hypothesis $(\mathrm{H})$ could be slightly relaxed, because our first main tool will be the next simple result.

Lemma 18 Let $\mu$ be a probability absolutely continuous with respect to the Lebesgue measure on $(0,+\infty)^{n}$, with $n \in \mathbb{N}^{*}$, and let $H$ be a measurable homogenous mapping from $(0,+\infty)^{n}$ to $(0,+\infty)$. Then the image of $\mu$ by $H$ does not contain atoms.

\section{Proof}

Let us recall that homogeneous means that there exists $\alpha>0$, called the degree, such that for any $x \in(0,+\infty)^{n}$ and any $t>0$, we have $H(t x)=t^{\alpha} H(x)$.

To prove the above result, it is sufficient to replace $\mu$ by $\nu$ the Lebesgue measure on $(0,+\infty)^{n}$, it does not matter that it is not a probability. Assume that $u \in(0,+\infty)$ is an atom of $H(\nu)$, namely that $\nu(\{x: H(x)=u\})>0$. Then for any $v \in(0,+\infty)$, we have

$$
\begin{aligned}
\nu(\{x: H(x)=v\}) & =\nu\left(\left\{x: H\left((u / v)^{1 / \alpha} x\right)=u\right\}\right) \\
& =\nu\left(\left\{(v / u)^{1 / \alpha} x: H(x)=u\right\}\right) \\
& =(v / u)^{n / \alpha} \nu(\{x: H(x)=u\}) \\
& >0
\end{aligned}
$$

This would imply that $\nu$ is not $\sigma$-finite, a contradiction.

The other elementary fact we will use is that if $Y$ and $Z$ are two independent random variables, the law of $Y$ containing no atom, then $\mathbb{P}[Y=Z]=0$ (where $\mathbb{P}$ will always denote the underlying probability).

We can now consider the first generical behavior pointed out in the introduction.

Proposition 19 Under assumption (H), we have

$$
\mathbb{P}\left[\exists 0 \leq l<k \leq N: c_{k, l} \in V\right]=0
$$

\section{Proof}

Let fix $0 \leq l<k \leq N$ and $v \in V \backslash\{0, N\}$. Of course, we just have to prove that $\mathbb{P}\left[c_{k, l}=v\right]=0$. Let $\mathcal{A}_{k-l}([v, N])$ be the subset of pseudo-partitions from $\mathcal{A}_{k-l}$ which form a quasi-partition of $[v, N]$. We define

$$
\Lambda_{k-l}([v, N])=\min _{A \in \mathcal{A}_{k-l}([v, N])} \Lambda(A)
$$

Indeed, if $c_{k, l}=v$, we have $\Lambda_{k-l}([v, N])=\Lambda_{k}$ and the minimum is attained in $A=\left(A_{l+1}^{(k)}, \ldots, A_{k}^{(k)}\right)$, as it is easily checked.

Let us define $\Lambda_{l}([0, v])$ in a symmetric way, where $\mathcal{A}_{k-l}([v, N])$ is replaced by $\mathcal{A}_{l}([0, v])$, the subset of pseudo-partitions from $\mathcal{A}_{l}$ which form a quasi-partition of $[0, v]$. Under the condition $c_{k, l}=v$, 
it appears equally that $\Lambda_{l}([0, v])=\Lambda_{k}$.

But as a random variable, $Y:=\Lambda_{k-l}([v, N])$ (respectively $Z:=\Lambda_{l}([0, v])$ only depends on $\left(b_{x}\right)_{v \leq x \leq N-1}$ (resp. $\left.\left(b_{x}\right)_{0 \leq x \leq v-1}\right)$ and is homogeneous of degree 1 in them. Thus Lemma 18 shows that under (H) the laws of $Y$ and $Z$ are without atom. Since furthermore $Y$ and $Z$ are independent, we get that $\mathbb{P}\left[c_{k, l}=v\right] \leq \mathbb{P}[Y=Z]=0$.

Our second and last generical result about birth and death processes follows from similar arguments. But we need first a preliminary observation.

Lemma 20 Let $A \in \mathcal{A}_{0}$ such that the restriction $f_{A}$ to $A \cap V$ of the minimizer $F_{A}$ (in the definition of $\left.\bar{\lambda}_{0}(A)\right)$ is maximum at two points, say $e^{-}$and $e^{+}=e^{-}+1$. Consider $\bar{V}_{+}:=\left[e^{+}, N\right]$ and the Dirichlet form $\mathcal{E}_{\bar{V}_{+}}$on $\mathcal{F}\left(\bar{V}_{+}\right)$given by

$$
\forall F \in \mathcal{F}\left(\bar{V}_{+}\right), \quad \mathcal{E}_{\bar{V}_{+}}(F):=\sum_{x \geq \in \llbracket e^{+}, N-1 \rrbracket} \int_{[x, x+1]}\left(F^{\prime}\right)^{2} d \lambda
$$

and define for any $B \in \mathcal{A}_{0}$ and $B \subset \bar{V}_{+}$,

$$
\lambda_{\bar{V}_{+}, 0}(B):=\inf _{f \in \mathcal{F}_{\bar{V}_{+}, 0}(B) \backslash\{0\}} \frac{\mathcal{E}_{\bar{V}_{+}}(f)}{\pi\left(f^{2}\right)}
$$

where $\mathcal{F}_{\bar{V}_{+}, 0}(B)$ is the set of functions from $\mathcal{F}\left(\bar{V}_{+}\right)$which vanish on $\bar{V}_{+} \backslash B$. Then we have

$$
\lambda_{\bar{V}_{+}, 0}\left(A \cap \bar{V}_{+}\right)=\bar{\lambda}_{0}(A)
$$

Of course, there is a symmetric result on the left of $e^{-}: \lambda_{\bar{V}_{-}, 0}\left(A \cap \bar{V}_{-}\right)=\bar{\lambda}_{0}(A)$, with a self-explaining notation.

\section{Proof}

One would have noticed that the objects introduced in this lemma are not really new. Let consider on $V_{+}:=\left\{e^{+}, e^{+}+1, \ldots, N\right\}$ and the generator $L_{+}$whose off-diagonal entries coincide with those of $L$. It is reversible with respect to $\pi_{+}$, the restriction to $V_{+}$of $\pi / \pi\left(V_{+}\right)$. Then $\lambda_{\bar{V}_{+}, 0}(\cdot)$ just corresponds to the functional $\bar{\lambda}_{0}(\cdot)$, but computed relatively to the reduced setting $\left(V_{+}, L_{+}, \pi_{+}\right)$ (in particular the continuous extension of $V_{+}$is $\bar{V}_{+}$).

Due to the assumption that $f_{A}\left(e^{+}\right)=f_{A}\left(e^{+}-1\right)$, we get that the relation of Lemma 2 is also satisfied if the underlying $L$ is replaced by $L_{+}$and $f_{\widetilde{A}}$ is replaced by its restriction to $V_{+}$. By a Perron-Frobenius argument, it follows that the latter is equally the restriction to $V_{+}$of the minimizer $F_{+, A \cap \bar{V}_{+}}$in the definition of $\lambda_{\bar{V}_{+}, 0}\left(A \cap \bar{V}_{+}\right)$, and that $\lambda_{\bar{V}_{+}, 0}\left(A \cap \bar{V}_{+}\right)=\bar{\lambda}_{0}(A)$.

We can now proceed to the

Proposition 21 Under assumption (H), we have

$$
\mathbb{P}\left[\exists 0<l<k \leq N: e_{k, l}^{-} \neq e_{k, l}^{+}\right]=0
$$

\section{Proof}

Again, let fix $0<l<k \leq N$ and $1<v<N$, we want to show that $\mathbb{P}\left[e_{k, l}^{-}=v-1, e_{k, l}^{+}=v\right]=0$. So let us assume that $e_{k, l}^{+}=v=e_{k, l}^{-}+1$. As in the proof of Lemma 20, we define $V_{+}:=\llbracket e_{k, l}^{+}, N \rrbracket$, we endow it with the natural generator $L_{+}$inherited from $L$, and we put a + in subscript of all notions relative to this setting. In particular we define $\Lambda_{+, k-l+1}$, which is smaller than the quantity $\Lambda_{k-l+1}([v, N])$ considered in proof of Proposition 19, because in the latter case we put a Dirichlet condition at $v$, while we are now rather imposing a Neumann condition. 
The important point is that the hypothesis $e_{k, l}^{+}=v=e_{k, l}^{-}+1$ implies that $\Lambda_{+, k-l+1}=\Lambda_{k}$. Indeed, through Lemma 20, we get that $\Lambda_{+, k-l+1} \leq \Lambda_{k}$ by considering the restriction to $[v, N]$ of the ordered quasi-partition $A^{(k)}$, which belongs to $\mathcal{A}_{+, k-l+1}$. But working symmetrically on $[0, v-1]$, it appears that $\Lambda_{-, l} \leq \Lambda_{k}$. So let an ordered $A_{-}:=\left(A_{-, 0}, A_{-, 1}, \ldots, A_{-, l}\right) \in \mathcal{A}_{-, l}$ be minimizing for $\Lambda_{-, l}$ and an ordered $A_{+}:=\left(A_{+, l}, A_{+, l+1}, \ldots, A_{+, k}\right) \in \mathcal{A}_{+, k-l+1}$ be minimizing for $\Lambda_{+, k-l+1}$. We define

$$
\forall 0 \leq j \leq k, \quad A_{j}^{\prime}:= \begin{cases}A_{-, j} & , \text { if } j<l \\ A_{-, l} \sqcup(v-1, v) \sqcup A_{+, l} & , \text { if } j=l \\ A_{+, j} & , \text { if } j>l\end{cases}
$$

One would have remarked that $v-1 \in A_{-, l}$ and $v \in A_{+, l}$, so in fact $A^{\prime}:=\left(A_{j}^{\prime}\right)_{0 \leq j \leq k}$ belongs to $\mathcal{A}_{0}$. Furthermore, we have

$$
\bar{\lambda}_{0}\left(A_{l}^{\prime}\right) \leq \bar{\lambda}_{-, 0}\left(A_{-, l}\right) \vee \bar{\lambda}_{+, 0}\left(A_{+, l}\right)
$$

as it is checked by considering the function $F \in \mathcal{F}_{0}\left(A_{l}^{\prime}\right)$ given by

$$
\forall x \in \bar{V}, \quad F(x):= \begin{cases}F_{-, A_{-, l}}(x) & , \text { if } x \leq v-1 \\ 1 & , \text { if } x \in(v-1, v) \\ F_{+, A_{+, l}}(x) & , \text { if } x \geq v\end{cases}
$$

(by our conventions and the facts that $F_{-, A_{-, l}}$ is nondecreasing on $[0, v-1]$ and $F_{+, A_{+, l}}$ is nonincreasing on $[v, N]$, we have $\left.F_{-, A_{-, l}}(v-1)=F_{+, A_{+}, l}(v)=1\right)$. Since furthermore, we have $\bar{\lambda}_{-, 0}\left(A_{-, j}\right)=\Lambda_{-}\left(A_{-}\right)$for $0 \leq j \leq l$, and $\bar{\lambda}_{+, 0}\left(A_{+, j}\right)=\Lambda_{+}\left(A_{+}\right)$for $l \leq j \leq k$, it appears that

$$
\begin{aligned}
\Lambda\left(A^{\prime}\right) & \leq \Lambda_{-}\left(A_{-}\right) \vee \Lambda_{+}\left(A_{+}\right) \\
& \leq \Lambda_{k}
\end{aligned}
$$

namely $A^{\prime}$ is a minimizing pseudo-partition for $\Lambda_{k}$. If follows that $\Lambda_{-}\left(A_{-}\right) \vee \Lambda_{+}\left(A_{+}\right)=\Lambda_{k}$ and necessarily

$$
\Lambda_{-}\left(A_{-}\right)=\Lambda_{k}=\Lambda_{+}\left(A_{+}\right)
$$

because $A^{\prime}$ must be well-balanced. So as we announced it above, the assumption $e_{k, l}^{+}=v=e_{k, l}^{-}+1$ implies that $\Lambda_{+, k-l+1}=\Lambda_{k}=\Lambda_{-, l}$. Now the end of the proof is similar to that of Proposition 19, since under $(\mathrm{H}), \Lambda_{+, k-l+1}$ and $\Lambda_{-, l}$ are independent random variables and homogeneous respectively in $\left(b_{x}\right)_{v \leq x<N}$ and $\left(b_{x}\right)_{0 \leq x \leq v-2}$.

We now leave the framework of birth and death processes to consider irreducible generators $L$ whose associated unoriented graph $(V, E)$ is a tree $\mathcal{T}$. They also admit a unique invariant probability $\pi$ which is positive and reversible. As above we will assume that it is fixed. To put a distribution on irreductible generators $L$ which are reversible with respect to $\pi$ and whose associated graph is $\mathcal{T}$, we choose an orientation of $\mathcal{T}$, i.e. any edge of $E$ gets an orientation and we call $\vec{E}$ their set. Since next hypothesis is an immediate extension of $(\mathrm{H})$, we give it the same name:

$$
\left\{\begin{array}{l}
\text { the birth rates }(L(x, y))_{(x, y) \in \vec{E}} \text { are independent and their laws are } \\
\text { absolutely continuous with respect to the Lebesgue measure on }(0,+\infty)
\end{array}\right.
$$

Of course, if $(x, y) \in \vec{E}$, we define $L(y, x)=\pi(x) L(x, y) / \pi(y)$. We have an extension of Proposition 19: 
Proposition 22 For $0 \leq k \leq N$, let $A^{(k)}:=\left(A_{0}^{(k)}, \ldots, A_{k}^{(k)}\right) \in \mathcal{A}_{k}$ be such that $\Lambda_{k}=\Lambda\left(A^{(k)}\right)$. Let $V_{3}\left(A^{(k)}\right):=V_{3} \cap \partial A^{(k)}$, with $\partial A^{(k)}:=\cup_{0 \leq l \leq k} \partial A_{l}^{(k)}$, and assume that $A^{(k)}$ has been chosen so that the cardinal of $V_{3}(A)$ is minimal among all minimizers of $\Lambda$ in $\mathcal{A}_{k}$. Then under assumption $(H)$, we have

$$
\mathbb{P}\left[\operatorname{card}\left(V_{3}\left(A^{(k)}\right)\right) \neq 0\right]=0
$$

The following arguments also show that if the minimizing $A^{(k)}$ was chosen among the well-balanced pseudo-partitions, as it is possible by Proposition 9, then the same conclusion holds.

\section{Proof}

Let $x \in V_{3}$ be a given vertex and denote by $x_{1}, \ldots, x_{n}$ its neighbours in $\mathcal{T}$, with $n \in \mathbb{N} \backslash\{0,1,2\}$. For $1 \leq i \leq n$, we consider $T_{i}$ the subtree rooted in $x$ going in the direction of $x_{i}$ : its vertex set is the subset of elements $y$ of $V$ whose unique nonintersecting path going from $x$ to $y$ has to pass through $x_{i}$. Let $\bar{T}_{i}$ be the union of edge-segment of $\bar{V}$ whose boundary vertices belong to $T_{i}$. For $0 \leq l \leq \operatorname{card}\left(T_{i}\right)-2$, we consider $\mathcal{A}_{l}\left(\bar{T}_{i}\right)$ the collection of pseudo-partitions from $\mathcal{A}_{l}$ whose elements are included into $\bar{T}_{i}$. Note they are in fact included into $\bar{T}_{i} \backslash\{x\}$, the interior of $T_{i}$, so that in what follows, one should keep in mind that a Dirichlet condition is put on $x$. Next, as in the proof of Proposition 19, we consider

$$
\Lambda_{l}\left(\bar{T}_{i}\right)=\min _{A \in \mathcal{A}_{l}\left(\bar{T}_{i}\right)} \Lambda(A)
$$

We say that $x$ is a splitting point if there $1 \leq i \neq j \leq n, 0 \leq l_{i} \leq \operatorname{card}\left(T_{i}\right)-2$ and $0 \leq l_{j} \leq$ $\operatorname{card}\left(T_{j}\right)-2$ such that $\Lambda_{l_{i}}\left(\bar{T}_{i}\right)=\Lambda_{l_{j}}\left(\bar{T}_{j}\right)$.

Now let $A^{(k)}$ be as in the previous proposition. The main step of its proof consists in showing that if $\partial A^{(k)} \cap V_{3} \neq \emptyset$, then one can find a splitting point.

Indeed, let assume that $x \in \partial A^{(k)} \cap V_{3}$ and let the subtrees rooted in $x$ be constructed as above. For $1 \leq i \leq n$, let $l_{i}+1 \in \llbracket 1, \operatorname{card}\left(T_{i}\right)-1 \rrbracket$ be the number of elements of $A^{(k)}$ included into $\bar{T}_{i}$. Considering these elements, we get that $\Lambda_{k} \geq \max _{1 \leq i \leq n} \Lambda_{l_{i}}\left(\bar{T}_{i}\right)$ and since the reverse inequality is always true (by considering the pseudo-partition formed by the union of the minimizing pseudopartitions for $\left.\Lambda_{l_{i}}\left(\bar{T}_{i}\right), 1 \leq i \leq n\right)$, it appears that

$$
\Lambda_{k}=\max _{1 \leq i \leq n} \Lambda_{l_{i}}\left(\bar{T}_{i}\right)
$$

If this maximum is attained at two indices $1 \leq i \neq j \leq n$, then $x$ is a splitting point. Otherwise, up to changes of indices, assume that the maximum is attained at $i=1$, that $A_{0}^{(k)}$ is included into $\bar{T}_{1}$ and that $x$ is a boundary point of $A_{0}^{(k)}$. Then starting from $x$, we can extend a little $A_{0}^{(k)}$ in the directions of $x_{2}, \ldots, x_{n}$ (in the same time reducing a little the other elements of $A^{(k)}$ which had $x$ as a boundary point), so that the slightly modified pseudo-partition we obtain in this way is still minimizing for $\Lambda_{k}$, but has less boundary points belonging to $V_{3}$, which is a contradiction with our choice of $A^{(k)}$.

The end of the proof is similar to that of Proposition 19, since for any $1 \leq i \leq n$ and $l \in$ $\llbracket 0, \operatorname{card}\left(T_{i}\right)-2 \rrbracket, \Lambda_{l}\left(\bar{T}_{i}\right)$ depends only on $(L(v, w))_{(v, w) \in \vec{E} \cap T_{i} \times T_{i}}$ and is homogeneous. Thus for any $x \in V_{3}$, any associated $1 \leq i \neq j \leq n$ and any $0 \leq l_{i} \leq \operatorname{card}\left(T_{i}\right)-2$ and $0 \leq l_{j} \leq \operatorname{card}\left(T_{j}\right)-2$, we have

$$
\mathbb{P}\left[\Lambda_{l_{i}}\left(\bar{T}_{i}\right)=\Lambda_{l_{j}}\left(\bar{T}_{j}\right)\right]=0
$$

Remark 23 We did not investigate measurability questions, in particular the existence of a measurable choice of $A^{(k)}$ as in the statement of the previous proposition (or at least that the mapping 
$\min \left\{\operatorname{card}\left(V_{3}(A)\right): A \in \mathcal{A}_{k}, \Lambda(A)=\Lambda_{k}\right\}$ is measurable). So to be more precise, the probability there should be understood as its completion with respect to negligible sets. Note nevertheless that (9) is rigorous, since the event $\left\{\Lambda_{l_{i}}\left(\bar{T}_{i}\right)=\Lambda_{l_{j}}\left(\bar{T}_{j}\right)\right\}$ is obviously measurable with respect to the rates $(L(x, y))_{(x, y) \in \vec{E}}$.

Proposition 22 associated with Proposition 7 and Proposition 10, shows that a.s. there exists a minimizing pseudo-partition $A^{(k)}$ for $\Lambda_{k}$ which is a well-balanced quasi-partition, for any $0 \leq k \leq$ $N$. But one can go further. Let $\mathcal{L}$ be the set of leaves of $\mathcal{T}$. If $A^{(k)} \in \mathcal{A}_{k}$ is minimizing for $\Lambda_{k}$ and satisfies $\partial A^{(k)} \cap V_{3}=\emptyset$, then we must have

$$
\mathcal{L} \subset \sqcup_{0 \leq l \leq k} A_{l}^{(k)}
$$

Indeed, for $0 \leq l \leq k$, let $\widetilde{A}_{l}^{(k)}:=A_{l}^{(k)} \cup\left\{x \in \mathcal{L} \cap \bar{A}_{l}^{(k)}\right\}$, where $\bar{A}_{l}^{(k)}$ is the closure of $A_{l}^{(k)}$. These sets are still open and connected, so $\widetilde{A}^{(k)}:=\left(\widetilde{A}_{l}^{(k)}\right)_{0 \leq l \leq k}$ belongs to $\mathcal{A}_{k}$ and since $\lambda_{0}\left(\widetilde{A}_{l}^{(k)}\right) \leq \lambda_{0}\left(A_{l}^{(k)}\right)$ for $0 \leq l \leq k, \widetilde{A}^{(k)}$ is also a minimizer for $\Lambda_{k}$. The relation $\partial \widetilde{A}^{(k)} \cap V_{3}=\emptyset$ equally holds, so $\widetilde{A}^{(k)}$ has to be well-balanced. But if (10) was not true, it would mean that for some $0 \leq l \leq k$, we have $\lambda_{0}\left(\widetilde{A}_{l}^{(k)}\right)<\lambda_{0}\left(A_{l}^{(k)}\right)$ and thus $\Lambda\left(\widetilde{A}^{(k)}\right)<\Lambda\left(A^{(k)}\right)$, a contradiction.

Then the procedure presented in Theorem 14 can also be applied to $A^{(k)}$, to construct a eigenvector $\psi_{k}$ corresponding to the eigenvalue $\Lambda_{k}$. One begins with one of the elements $A_{l}^{(k)}$ of $A^{(k)}$ which contains a leaf from $\mathcal{L}$. On $A_{l}^{(k)}$, one takes $\psi_{k}=f_{A_{l}^{(k)}}$, the restriction of $F_{A_{l}^{(k)}}$ on $V \cap A_{l}^{(k)}$. Next one considers another domain $A_{l^{\prime}}^{(k)}$ admitting a boundary point $x$ in common with $A_{l}^{(k)}$ (since $\bar{V}$ is a continuous tree there is only one such a common boundary point between $A_{l^{\prime}}^{(k)}$ and $\left.A_{l}^{(k)}\right)$. Then one can find a negative factor $r$ such that by taking $\psi_{k}=r f_{A_{l^{\prime}}^{(k)}}$ on $A_{l^{\prime}}^{(k)}$, we get $L\left[\psi_{k}\right]=\Lambda_{k} \psi_{k}$ on $V \cap\left(A_{l}^{(k)} \sqcup\{x\} \sqcup A_{l^{\prime}}^{(k)}\right)$, except on the leaves of this subtree which do not belong to $\mathcal{L}$ (because of (10)). But this construction can be iterated, by choosing one of the boundary point of $A_{l}^{(k)} \sqcup\{x\} \sqcup A_{l^{\prime}}^{(k)}$ which is not a leaf from $\mathcal{L}$. Having a closer look at this construction, it appears that one always gets the same function $\psi_{k}$, up to a factor and indeed the obtained function only depends on the initial choice of $A_{l}^{(k)}$. Thus we can normalize $\psi_{k}$ by fixing a root among the leaves (say 0 , up to reordering of $V$ ) and by beginning the previous construction with the domain $A_{l}^{(k)}$ containing 0 . Next result shows that $\left(\Lambda_{k}, \psi_{k}\right)_{0 \leq k \leq N}$ is a.s. a spectral decomposition of $L$ under $(\mathrm{H})$.

Proposition 24 Under $(H)$, we have a.s. that all the $\Lambda_{k}$, for $0 \leq k \leq N$, are distinct. It follows that $\Lambda_{k}=\lambda_{k}$ for all $0 \leq k \leq N$.

\section{Proof}

Let $0<k<N$ be fixed and assume that $\Lambda_{k}=\Lambda_{k+1}$. Under (H) we can a.s. find a well-balanced quasi-partition $A^{(k)}$ (respectively $A^{(k+1)}$ ) which is minimizing for $\Lambda_{k}$ (resp. $\Lambda_{k+1}$ ). One can put a tree structure on $\left\{A_{l}^{(k)}: 0 \leq l \leq k\right\}$ by saying that $A_{l}^{(k)}$ and $A_{l^{\prime}}^{(k)}$ are neighbours if they have a boundary point in common. Up to a change of indices, assume that $A_{0}^{(k)}$ is a leaf of this tree. Then, because of (10), it admits a unique boundary point $x_{0} \in \bar{V}$ (note that we only considered non-trivial cases where $k>0$ ) and we have $x_{0} \notin \mathcal{L}$. Let denote by $\mathcal{L}_{0}$ the set of leaves from $\mathcal{L}$ belonging to $A_{0}^{(k)}$. It appears by our choice of $A_{0}^{(k)}$, that $\mathcal{L}_{0} \neq \emptyset$, so let $x_{1} \in \mathcal{L}_{0}$. Resorting again to (10) applied to $A^{(k+1)}$, there exist $0 \leq l \leq k+1$ such that $x_{1} \in A_{l}^{(k+1)}$ and up to a change of indices, assume that $l=0$. We are going to show that

$$
A_{0}^{(k+1)}=A_{0}^{(k)}
$$

Indeed, assume that $A_{0}^{(k)} \backslash A_{0}^{(k+1)}$ is not empty. Then one of its connected components must either contain an element of $\mathcal{L}_{0}$ or admits $x_{0}$ as boundary point. The latter situation implies 
that $A_{0}^{(k+1)}$ is strictly included into $A_{0}^{(k)}$ and so by well-balancedness we would conclude that $\Lambda_{k+1}>\Lambda_{k}$, in contradiction with our working assumption. We can now assume that $x_{0}$ belongs to the closure of $A_{0}^{(k+1)}$ and so we are in the former situation where $\left(A_{0}^{(k)} \backslash A_{0}^{(k+1)}\right) \cap \mathcal{L}_{0} \neq \emptyset$. Let $x_{2} \in\left(A_{0}^{(k)} \backslash A_{0}^{(k+1)}\right) \cap \mathcal{L}_{0}$, there exists $0 \leq l \leq k+1$, with $l \neq 0$, such that $x_{2} \in A_{l}^{(k+1)}$. We must have that $A_{l}^{(k+1)}$ is strictly included into $A_{0}^{(k)}$ and we are thus led to a new contradiction. So it appears that $A_{0}^{(k)} \subset A_{0}^{(k+1)}$. This inclusion cannot be strict, otherwise our assumption $\Lambda_{k}=\Lambda_{k+1}$ would be broken again. This ends the proof of the validity of (11).

But this procedure can be iterated on $\bar{V} \backslash A_{0}^{(k)}$ (with the slight difference that a Dirichlet condition is put on $x_{0}$, but the above arguments can be extended to this situation), with respect to the collections $\left(A_{l}^{(k)}\right)_{1 \leq l \leq k}$ and $\left(A_{l}^{(k+1)}\right)_{1 \leq l \leq k+1}$. The conclusion is that there exist $1 \leq l \leq k$ and $1 \leq l^{\prime} \leq k+1$ such that $A_{l}^{(k)}=A_{l^{\prime}}^{(k+1)}$. In this way we show that any $A_{l}^{(k)}$, for $0 \leq l \leq k$, is equal to some $A_{l^{\prime}}^{(k+1)}$, which means there exists $0 \leq m \leq k+1$ such that $A_{m}^{(k+1)}=\emptyset$. This contradiction implies that it is impossible that $\Lambda_{k}=\Lambda_{k+1}$, so that the $\Lambda_{k}$, for $0 \leq k \leq N$ are all distinct. But we have seen they are eigenvalues of $L$, which are thus all attained by the above constructions.

Proposition 24 shows that under its assumption, the minimizing well-balanced quasi-partitions are uniquely determined (up to a change of indices): they correspond to the nodal domains of the piecewise affine extensions to $\bar{V}$ of the eigenvectors, as it was explained after the proof of Proposition 12. From now on, we will refer to these quasi-partitions by $\left(A_{l}^{(k)}\right)_{0 \leq l \leq k}$ (ordered by their minimal elements, say).

Under $(\mathrm{H})$, more a.s. informations can be deduced for the eigenvectors $\left(\psi_{k}\right)_{0 \leq k \leq N}$, by adapting the arguments given in the setting of birth and death processes (Proposition 19 for the first point and Lemma 20 and Proposition 21 for the second one):

- The eigenvectors do not vanish: $\psi_{k}(x) \neq 0$ for any $0 \leq k \leq N$ and any $x \in V$.

- For any $0 \leq k \leq N$ and $\{x, y\} \in E$, we have $\psi_{k}(x) \neq \psi_{k}(y)$.

But we can no longer deduce from this property that for any $0<k \leq N$ and $0 \leq l \leq k,\left|\psi_{k}\right|$ attains its maximum on $A_{l}^{(k)} \cap V$ at a unique point. In fact it is not true that a.s. there is a unique local extremum on $A_{l}^{(k)} \cap V$ (namely a point $x \in A_{l}^{(k)} \cap V$ such that $\left|\psi_{k}(x)\right|>\left|\psi_{k}(y)\right|$ for any neighbour $y \in A_{l}^{(k)} \cap V$ of $x$ ), see for instance lemma 27 in next section. To finish this section, we prove a result announced in section 2 .

Theorem 25 Let $L$ be any irreducible generator whose associated graph is a tree. Then for any $0 \leq k \leq N$, we have

$$
\Lambda_{k}=\lambda_{k}
$$

\section{Proof}

Again let the tree $\mathcal{T}$ and the reversible probability $\pi$ be fixed, so that the quantities $\Lambda_{k}$ and $\lambda_{k}$, for $0 \leq k \leq N$, can be seen as functions of the parameters $(L(x, y))_{(x, y) \in \vec{E}} \in\left(\mathbb{R}_{+}^{*}\right)^{\vec{E}}$. We begin by showing that these functions are continuous.

Indeed, let $\left(L^{\prime}(x, y)\right)_{(x, y) \in \vec{E}} \in\left(\mathbb{R}_{+}^{*}\right)^{\vec{E}}$ be another collection of parameters such that for some $a>0$,

$$
\forall(x, y) \in \vec{E}, \quad L(x, y) \leq a L^{\prime}(x, y)
$$

Then we have that the corresponding Dirichlet forms satisfy $\mathcal{E} \leq a \mathcal{E}^{\prime}$ and it follows straightfowardly that

$$
\forall 0 \leq k \leq N, \quad\left\{\begin{array}{l}
\Lambda_{k} \leq a \Lambda_{k}^{\prime} \\
\lambda_{k} \leq a \lambda_{k}^{\prime}
\end{array}\right.
$$


Optimizing in $a$, we deduce from these relations that for $0<k \leq N$, we have

$$
\begin{aligned}
\ln \left(\Lambda_{k}\right)-\ln \left(\Lambda_{k}^{\prime}\right) & \leq \max _{(x, y) \in \vec{E}} \ln (L(x, y))-\ln \left(L^{\prime}(x, y)\right) \\
\ln \left(\lambda_{k}\right)-\ln \left(\lambda_{k}^{\prime}\right) & \leq \max _{(x, y) \in \vec{E}} \ln (L(x, y))-\ln \left(L^{\prime}(x, y)\right)
\end{aligned}
$$

and by symmetry that

$$
\begin{aligned}
\left|\ln \left(\Lambda_{k}\right)-\ln \left(\Lambda_{k}^{\prime}\right)\right| & \leq \max _{(x, y) \in \vec{E}}\left|\ln (L(x, y))-\ln \left(L^{\prime}(x, y)\right)\right| \\
\left|\ln \left(\lambda_{k}\right)-\ln \left(\lambda_{k}^{\prime}\right)\right| & \leq \max _{(x, y) \in \vec{E}}\left|\ln (L(x, y))-\ln \left(L^{\prime}(x, y)\right)\right|
\end{aligned}
$$

The wanted continuity properties follow at once.

But we have seen that the relations $\Lambda_{k}=\lambda_{k}$, for $0 \leq k \leq N$, are verified a.s. if $(L(x, y))_{(x, y) \in \vec{E}}$ is distributed according to the tensor product of the exponential law of parameter 1, in particular they are satisfied on a dense subset of $\left(\mathbb{R}_{+}^{*}\right)^{\vec{E}}$. The previous continuity properties then allow to extend these relations $\Lambda_{k}=\lambda_{k}$ over the whole set $\left(\mathbb{R}_{+}^{*}\right) \vec{E}$, namely for any irreducible generator $L$ whose reversible probability is $\pi$ and whose associated graph is $\mathcal{T}$.

Remark 26 Even when the ordered pseudo-partitions are unique, they are not necessarily continuous as functions of the rates $(L(x, y))_{(x, y) \in \vec{E}}$, for instance in the sense of Hausdorff topology. Coming back to Example 8, let us diminish a little the edge $[0,1]$ by considering for small $\epsilon>0$,

$$
L=\left(\begin{array}{cccc}
-10-\epsilon & 4+\epsilon & 4 & 2 \\
4+\epsilon & -4 & 0 & 0 \\
4 & 0 & -4 & 0 \\
2 & 0 & 0 & -2
\end{array}\right)
$$

Then we get that

$$
\lim _{\epsilon \rightarrow 0_{+}} A^{(1)}(\epsilon)=([1,0] \cup[0,3],[2,0))
$$

which is different from $A^{(1)}(0)=([1,0),[2,0))$.

\section{$5 \quad$ Spectral gap eigenfunctions on trees}

We will investigate here the shape of the eigenfunctions corresponding to the spectral gap of generators $L$ whose associated graph is a tree $\mathcal{T}$. More precisely, our goal is to prove the next two results.

- There exists a center point $x_{0} \in \bar{V}$ in the following sense:

- If $x_{0} \notin V$, let us write $\bar{V} \backslash\left\{x_{0}\right\}=A_{1} \sqcup A_{2}$, with $A_{1}, A_{2} \in \mathcal{A}_{0}$. Then the eigenspace corresponding to $\lambda_{1}$ is one-dimensional and is generated by the function $\varphi_{1}$ given by

$$
\forall x \in V, \quad \varphi_{1}(x):= \begin{cases}f_{1}(x) & , \text { if } x \in V \cap A_{1} \\ r f_{2}(x) & \text {, if } x \in V \cap A_{2}\end{cases}
$$

for an appropriate choice of $r<0$, where for $i=1,2, f_{i}$ designates the restriction to $V \cap A_{i}$ of the minimizer $F_{A_{i}}$ for $\bar{\lambda}_{0}\left(A_{i}\right)$. 
- If $x_{0} \in V$, let us write $\bar{V} \backslash\left\{x_{0}\right\}=\sqcup_{1 \leq i \leq n} A_{i}$ with $A_{i} \in \mathcal{A}_{0}$ for $0 \leq i \leq n$. Assume they are ordered such that the finite sequence $\left(\bar{\lambda}_{0}\left(A_{i}\right)\right)_{1 \leq i \leq n}$ is nondecreasing and let $1 \leq m \leq n$ be the number of these sets with the smallest $\bar{\lambda}_{0}$, so that $\bar{\lambda}_{0}\left(A_{1}\right)=\cdots=\bar{\lambda}_{0}\left(A_{m}\right)>\bar{\lambda}_{0}\left(A_{m+1}\right) \geq \cdots \geq \bar{\lambda}_{0}\left(A_{n}\right)$ (we will see that $m \geq 2$ ). For $1 \leq i \leq m$, let $x_{i}$ be the neighbour of $x_{0}$ belonging to $A_{i}$ and denote by $f_{i}$ the restriction of $F_{A_{i}}$ on $V \cap A_{i}$, extended by 0 on $V \backslash A_{i}$. Then the eigenspace associated to $-\lambda_{1}$ is

$$
\left\{\varphi \in \mathcal{F}(V): \varphi=\sum_{1 \leq i \leq m} r_{i} f_{i}, \text { with } \sum_{1 \leq i \leq m} r_{i} f_{i}\left(x_{i}\right)=0\right\}
$$

which is of dimension $m-1$ (recall that $f_{i}\left(x_{i}\right)>0$ ).

- Let $\varphi$ be any eigenfunction associated to $-\lambda_{1}$. Then there exists a partial order $\preceq$ on $V$ compatible with $\mathcal{T}$ (this means that, on one hand, for any edge $\{x, y\} \in E$, we have either $x \preceq y$ or $y \preceq x$ and on the other hand, for any $x, y, z \in V$, if $x \preceq y \preceq z$, then $y$ must lay on the nonintersecting path going from $x$ to $z$ ), such that $\varphi$ is nondecreasing. But contrary to the center point $x_{0}$, this order is not unique and depends on the chosen eigenfunction $\varphi$.

We start with the existence of the center point. To find it, let us consider $A^{(1)}:=\left(A_{0}^{(1)}, A_{1}^{(1)}\right)$ a minimizing pseudo-partition for $\Lambda_{1}$. By Proposition 7 , it is necessarily well-balanced. Furthermore any boundary point of $A_{0}^{(1)}$ must also be a boundary point of $A_{1}^{(1)}$, otherwise we could extend a little $A_{0}^{(1)}$ to be led to a contradiction. Since $\mathcal{T}$ is a tree, this common boundary point is unique, let us call it $x_{0}$. We now consider two cases.

- If $x_{0} \notin V$, then by Proposition 10, $A^{(1)}$ is also a quasi-partition and as in Theorem 14 or in the discussion after Remark 23, we can use it to construct an eigenvector $\varphi_{1}$ of the form (12) associated to $-\lambda_{1}=-\Lambda_{1}$ (by Theorem 25). Next consider any other eigenvector $\varphi$ associated to $-\lambda_{1}$. Let $A_{1}, \ldots, A_{p}$ be the $p \geq 2$ nodal domains of its affine extension to $\bar{V}$. As it was explained after the proof of Proposition 12, we have $\bar{\lambda}_{0}\left(A_{i}\right)=\lambda_{1}$ for $1 \leq i \leq p$. But since $A^{(1)}$ is a quasi-partition formed of two (continuous) subtrees, necessarily there exist $i \in\{0,1\}$ and $j \in\{1, \ldots, p\}$ such that $A_{j} \subset A_{i}^{(1)}$. Indeed we must have $A_{j}=A_{i}^{(1)}$, otherwise we would have $\bar{\lambda}_{0}\left(A_{j}\right)>\bar{\lambda}_{0}\left(A_{i}^{(1)}\right)$. The same kind of argument implies next that $p=2$ and that $A^{(1)}=\left(A_{1}, A_{2}\right)$, up to a change of order. It follows that up to factors, the restrictions of $\varphi$ to $A_{1}$ and $A_{2}$ coincide with the restrictions of the minimizers $F_{A_{0}^{(1)}}$ and $F_{A_{0}^{(1)}}$ to $V \cap A_{0}^{(1)}$ and $V \cap A_{1}^{(1)}$, respectively. By the uniqueness of the appropriate choice of $r$ in (12), we get that $\varphi$ is proportional to $\varphi_{1}$ and it appears that $-\lambda_{1}$ is of multiplicity one.

- If $x_{0} \in V$, let construct $A_{1}, \ldots, A_{n}$ and $1 \leq m \leq n$ as in the beginning of this section. Obviously there exist $1 \leq i \neq j \leq n$ such that $A_{0}^{(1)} \subset A_{i}$ and $A_{1}^{(1)} \subset A_{j}$. In fact we must even have equality, otherwise it would be possible to extend a little $A_{0}^{(1)}$ or $A_{1}^{(1)}$. Then by definition of $A^{(1)}$, we have $\Lambda\left(A^{(1)}\right)=\Lambda\left(\left(A_{1}, A_{2}\right)\right)$ and since any minimizing pseudo-partition for $\Lambda_{1}$ is well-balanced, it it follows that $\bar{\lambda}_{0}\left(A_{1}\right)=\bar{\lambda}_{0}\left(A_{2}\right)=\Lambda_{1}=\lambda_{1}$, in particular $m \geq 2$.

Let $\varphi$ be a function belonging to the space defined in (13). Then for any $1 \leq i \leq m$ and any $x \in A_{i}$, we have, by virtue of the characterization given in Lemma 2

$$
\begin{aligned}
L[\varphi](x) & =-\bar{\lambda}_{0}\left(A_{i}\right) \varphi(x) \\
& =-\lambda_{1} \varphi(x)
\end{aligned}
$$

The condition $\sum_{1 \leq i \leq m} r_{i} f_{i}\left(x_{i}\right)=0$ insures that

$$
\begin{aligned}
L[\varphi]\left(x_{0}\right) & =0 \\
& =-\lambda_{1} \varphi\left(x_{0}\right)
\end{aligned}
$$


so the relation $L[\varphi]=-\lambda_{1} \varphi$ holds everywhere on $V$ and $\varphi$ is an eigenvector associated to $-\lambda_{1}$. Conversely, let $\varphi$ be any eigenvector associated to $-\lambda_{1}$ and consider the nodal domains of its affine extension to $\bar{V}$, say $D_{1}, \ldots, D_{p}$, with $p \geq 2$. As it has already been observed several times, we must have $\lambda_{1}=\bar{\lambda}_{0}\left(D_{1}\right)=\cdots=\bar{\lambda}_{0}\left(D_{p}\right)$. By the geometry of the tree $\mathcal{T}$ rooted in $x_{0}$, at least one of the $D_{i}$, with $1 \leq i \leq p$ is included into one of the $A_{j}$, with $1 \leq j \leq n$. If $m<j \leq n$, we would have $\bar{\lambda}_{0}\left(D_{i}\right) \geq \bar{\lambda}_{0}\left(A_{j}\right)>\lambda_{1}$, a contradiction. So $1 \leq j \leq m$ and $D_{i}$ must be equal to $A_{j}$, otherwise we would end up with a contradiction again. This implies that $x_{0}$ is a boundary point of $D_{i}$, so each of the $D_{i^{\prime}}$, with $1 \leq i^{\prime} \neq i \leq$, is included into some $A_{j^{\prime}}$, with $1 \leq j^{\prime} \neq j \leq n$. The previous arguments show that these indices $j^{\prime}$ must be less or equal to $m$ and that in fact we have equality, not only inclusion, between those sets. Thus $p \leq m$ and there is a one-to-one mapping $\sigma: \llbracket 1, p \rrbracket \rightarrow \llbracket 1, m \rrbracket$ such that for any $1 \leq i \leq p, D_{i}=A_{\sigma(i)}$. It is now easy to deduce that $\varphi$ belongs to the set (13), since we know that $\varphi\left(x_{0}\right)=0$. This ends the proof that (13) coincides with the eigenspace associated with $-\lambda_{1}$.

To study the monotonicity properties of the eigenvectors associated with $-\lambda_{1}$, we need to come back to minimizer functions for $\bar{\lambda}_{0}$.

Lemma 27 Let $A \in \mathcal{A}_{0}$ admitting a unique boundary point $x_{0}$. The subset $A \sqcup\left\{x_{0}\right\}$ can be seen as a continuous tree rooted in $x_{0}$ and this endows $A \sqcup\left\{x_{0}\right\}$ with a partial order $\unlhd$ by deciding that for any $x, y \in A \sqcup\left\{x_{0}\right\}, x \unlhd y$ if and only if $x$ is on the (continuous) nonintersecting path going from $x_{0}$ to $y$. Then the restriction to $A \sqcup\left\{x_{0}\right\}$ of the minimizer $F_{A}$ is increasing.

\section{Proof}

Since we know that $F_{A}$ is affine on each of the edge-segment (and on $\left[x_{0}, x_{1}\right]$, where $x_{1}$ is the closest element to $x_{0}$ in $V \cap A$, note also that $F_{A}\left(x_{1}\right)>0$, so we already get that $F$ is increasing on $\left.\left[x_{0}, x_{1}\right]\right)$, it is sufficient to prove that for any $\{x, y\} \in E$ with $x, y \in V \cap A$, we have $F_{A}(x)<F_{A}(y)$ if $x \triangleleft y$. We begin by showing that $F_{A}(x) \leq F_{A}(y)$. Indeed, if it is not true, consider the function $f$ defined by

$$
\forall z \in(V \cap A) \sqcup\left\{x_{0}\right\}, \quad f(z):= \begin{cases}F_{A}(z) & , \text { if } z \unlhd x \\ F_{A}(z)+2\left(F_{A}(x)-F_{A}(y)\right) & , \text { if } y \unlhd z\end{cases}
$$

Next we extend affinely (on each edge-segment and on $\left[x_{0}, x_{1}\right]$ ) $f$ into $F$ on $A \sqcup\left\{x_{0}\right\}$. Let also $F$ vanish outside $A$, so that $F \in \mathcal{F}_{0}(A)$. It appears that $\overline{\mathcal{E}}(F)=\overline{\mathcal{E}}\left(F_{A}\right)$, but since we already know that $F_{A} \geq 0$, we have $\pi\left(F^{2}\right)>\pi\left(F_{A}^{2}\right)$. This is in contradiction with the definition of $F_{A}$ as a minimizer. Thus we get that $F_{A}(x) \leq F_{A}(y)$ and it follows that $F_{A}$ is nondecreasing on $A \sqcup\left\{x_{0}\right\}$. The fact that it is indeed increasing is a consequence of Proposition 11.

We can now investigate the second feature of eigenvectors associated to spectral gap mentioned in the beginning of this section. Again we consider two cases.

- If the center point $x_{0}$ does not belong to $V$. It is enough to consider the eigenvector $\varphi_{1}$ defined in (12). Let $\preceq$ be the binary relation which coincides on $\left(V \cap A_{1}\right) \sqcup\left\{x_{0}\right\}$ (respectively on $\left.\left(V \cap A_{2}\right) \sqcup\left\{x_{0}\right\}\right)$ with the (resp. reverse) partial order $\unlhd$ presented in the previous lemma with $A=A_{1}$ (resp. $A=A_{2}$ ). We complete $\preceq$ into a partial order by asking that for any $x \in V \cap A_{2}$ and any $y \in V \cap A_{1}, x \preceq y$. It is easy to verify that $\preceq$ is compatible with $\mathcal{T}$ and that $\varphi_{1}$ is increasing with respect to it.

- If the center point $x_{0}$ belongs to $V$. Let $\varphi$ be a function from the eigenspace (13). We denote $I_{+}:=\left\{1 \leq i \leq m: r_{i}>0\right\}, I_{-}:=\left\{1 \leq i \leq m: r_{i}<0\right\}$ and $I_{0}:=\left\{1 \leq i \leq m: r_{i}=0\right\} \sqcup \llbracket m+1, n \rrbracket$. Let $\preceq$ be the binary relation which coincides on $\left(V \cap A_{i}\right) \sqcup\left\{x_{0}\right\}$, for $i \in I_{+} \sqcup I_{0}$ (respectively for $i \in I_{-}$) with the (resp. reverse) partial order $\unlhd$ presented in Lemma 27 with $A=A_{i}$. We complete $\preceq$ into a partial order by asking that for any $x \in V \cap A_{i}$ and any $y \in V \cap A_{j}$, with $i \in I_{-}$and $j \in I_{+} \sqcup I_{0}$, we have $x \preceq y$. Then $\preceq$ is compatible with $\mathcal{T}$ and $\varphi_{1}$ is nondecreasing with respect 
to it. But in general $\preceq$ is not the unique partial order which satisfies these properties with respect to $\varphi$, because on $\left(V \cap A_{i}\right) \sqcup\left\{x_{0}\right\}$ with $i \in I_{0}$, we could also have chosen the reverse partial order (one would have noticed that $\varphi$ vanishes on $\left\{x_{0}\right\} \sqcup \cup_{i \in I_{0}} V \cap A_{i}$ ).

\section{Aknowledgments:}

This paper was motivated by discussions with Persi Diaconis, Susan Holmes and Julia Salzman, who were interested in another approach to the subject and encouraged me to write down these notes. In particular, Julia Salzman [18] has a simple proof of the fact that the generator of a birth and death process is totally positive, up to the addition of a factor of the identity. I also thank Persi Diaconis' CNRS Chaire d'excellence and the Laboratoire J. A. Dieudonné (UMR 6621) in Nice, for their support and hospitality.

\section{References}

[1] David Aldous and Jim Fill. Reversible Markov chains and random walks on graphs. Monograph in preparation, available on the web site: http://www.stat.berkeley.edu/ aldous/RWG/book.html, 1994-2002.

[2] Türker Bıyıkoğlu. A discrete nodal domain theorem for trees. Linear Algebra Appl., 360:197205, 2003.

[3] Jeff Cheeger. A lower bound for the smallest eigenvalue of the Laplacian. In Problems in analysis (Papers dedicated to Salomon Bochner, 1969), pages 195-199. Princeton Univ. Press, Princeton, N. J., 1970.

[4] $\mathrm{Mu}-\mathrm{Fa}$ Chen and Feng-Yu Wang. Estimation of spectral gap for elliptic operators. Trans. Amer. Math. Soc., 349(3):1239-1267, 1997.

[5] Mufa Chen. Analytic proof of dual variational formula for the first eigenvalue in dimension one. Sci. China Ser. A, 42(8):805-815, 1999.

[6] Mufa Chen. Variational formulas and approximation theorems for the first eigenvalue in dimension one. Sci. China Ser. A, 44(4):409-418, 2001.

[7] R. Courant and D. Hilbert. Methods of mathematical physics. Vol. I. Interscience Publishers, Inc., New York, N.Y., 1953.

[8] Pierre Del Moral and L. Miclo. Particle approximations of Lyapunov exponents connected to Schrödinger operators and Feynman-Kac semigroups. ESAIM Probab. Stat., 7:171-208 (electronic), 2003.

[9] Persi Diaconis and James Allen Fill. Strong stationary times via a new form of duality. Ann. Probab., 18(4):1483-1522, 1990.

[10] Sergey Fomin and Andrei Zelevinsky. Total positivity: tests and parametrizations. Math. Intelligencer, 22(1):23-33, 2000.

[11] Joel Friedman. Some geometric aspects of graphs and their eigenfunctions. Duke Math. J., 69(3):487-525, 1993.

[12] Masatoshi Fukushima, Yōichi Ōshima, and Masayoshi Takeda. Dirichlet forms and symmetric Markov processes, volume 19 of de Gruyter Studies in Mathematics. Walter de Gruyter \& Co., Berlin, 1994.

[13] F.R. Gantmacher and M.G. Krein. Sur les matrices complétement non négatives et oscillatoires. Compositio Mathematica, 4:445-470, 1937.

[14] S. Karlin and J. L. McGregor. The differential equations of birth-and-death processes, and the Stieltjes moment problem. Trans. Amer. Math. Soc., 85:489-546, 1957. 
[15] Samuel Karlin. Total positivity. Vol. I. Stanford University Press, Stanford, Calif, 1968.

[16] Gregory F. Lawler and Alan D. Sokal. Bounds on the $L^{2}$ spectrum for Markov chains and Markov processes: a generalization of Cheeger's inequality. Trans. Amer. Math. Soc., 309(2):557-580, 1988.

[17] L. Miclo. Monotonicité des fonctions extrémales pour les inégalités de type Sobolev logarithmiques en dimension 1. Preprint, available on http://hal.ccsd.cnrs.fr/ccsd-00019571, 2005.

[18] Julia Salzman. Personal communication and P.H.D. thesis in preparation, 2007.

[19] Isac Schoenberg. Über variationsvermindernde lineare Transformationen. Math. Z., 32(1):321$328,1930$.

miclo@latp.univ-mrs.fr

Laboratoire d'Analyse, Topologie, Probabilités

Centre de Mathématiques et Informatique

Université de Provence

39, rue Frédéric Joliot-Curie

13453 Marseille cedex 13, France 